NIST Special Publication 1500-7r1

\title{
NIST Big Data Interoperability Framework: \\ Volume 7, Standards Roadmap
}




\section{NIST Special Publication 1500-7r1}

\section{NIST Big Data Interoperability Framework: Volume 7, Standards Roadmap \\ Version 2}

NIST Big Data Public Working Group (NBD-PWG)

Standards Roadmap Subgroup

Information Technology Laboratory

National Institute of Standards and Technology

Gaithersburg, MD 20899

This publication is available free of charge from:

https://doi.org/10.6028/NIST.SP.1500-7r1

June 2018

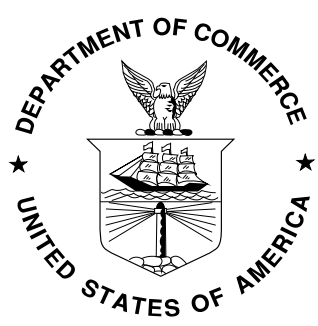

U.S. Department of Commerce Wilbur L. Ross, Jr., Secretary

National Institute of Standards and Technology Walter Copan, NIST Director and Undersecretary of Commerce for Standards and Technology 
National Institute of Standards and Technology (NIST) Special Publication 1500-7r1 Natl. Inst. Stand. Technol. Spec. Publ. 1500-7r1, 71 pages (June 2018) CODEN: NSPUE2

This publication is available free of charge from: https://doi.org/10.6028/NIST.SP.1500-7r1

Certain commercial entities, equipment, or materials may be identified in this document to describe an experimental procedure or concept adequately. Such identification is not intended to imply recommendation or endorsement by NIST, nor is it intended to imply that the entities, materials, or equipment are necessarily the best available for the purpose.

There may be references in this publication to other publications currently under development by NIST in accordance with its assigned statutory responsibilities. The information in this publication, including concepts and methodologies, may be used by Federal agencies even before the completion of such companion publications. Thus, until each publication is completed, current requirements, guidelines, and procedures, where they exist, remain operative. For planning and transition purposes, Federal agencies may wish to closely follow the development of these new publications by NIST.

Organizations are encouraged to review all publications during public comment periods and provide feedback to NIST. All NIST publications are available at http://www.nist.gov/publication-portal.cfm.

\title{
Comments on this publication may be submitted to Wo Chang
}

\author{
National Institute of Standards and Technology \\ Attn: Wo Chang, Information Technology Laboratory \\ 100 Bureau Drive (Mail Stop 8900) Gaithersburg, MD 20899-8930 \\ Email: SP1500comments@nist.gov
}




\section{Reports on Computer Systems Technology}

The Information Technology Laboratory (ITL) at NIST promotes the U.S. economy and public welfare by providing technical leadership for the Nation's measurement and standards infrastructure. ITL develops tests, test methods, reference data, proof of concept implementations, and technical analyses to advance the development and productive use of information technology (IT). ITL's responsibilities include the development of management, administrative, technical, and physical standards and guidelines for the cost-effective security and privacy of other than national security-related information in Federal information systems. This document reports on ITL's research, guidance, and outreach efforts in IT and its collaborative activities with industry, government, and academic organizations.

\section{Abstract}

Big Data is a term used to describe the large amount of data in the networked, digitized, sensor-laden, information-driven world. While opportunities exist with Big Data, the data can overwhelm traditional technical approaches and the growth of data is outpacing scientific and technological advances in data analytics. To advance progress in Big Data, the NIST Big Data Public Working Group (NBD-PWG) is working to develop consensus on important, fundamental concepts related to Big Data. The results are reported in the NIST Big Data Interoperability Framework (NBDIF) series of volumes. This volume, Volume 7, contains summaries of the work presented in the other six volumes, an investigation of standards related to Big Data, and an inspection of gaps in those standards.

\section{Keywords}

Big Data; Big Data Application Provider; Big Data characteristics; Big Data Framework Provider; Big Data standards; Big Data taxonomy; Data Consumer; Data Provider; Management Fabric; reference architecture; Security and Privacy Fabric; System Orchestrator; use cases 


\section{Acknowledgements}

This document reflects the contributions and discussions by the membership of the NBD-PWG, cochaired by Wo Chang (NIST ITL), Bob Marcus (ET-Strategies), and Chaitan Baru (San Diego Supercomputer Center; National Science Foundation). For all versions, the Subgroups were led by the following people: Nancy Grady (SAIC), Natasha Balac (San Diego Supercomputer Center), and Eugene Luster (R2AD) for the Definitions and Taxonomies Subgroup; Geoffrey Fox (Indiana University) and Tsegereda Beyene (Cisco Systems) for the Use Cases and Requirements Subgroup; Arnab Roy (Fujitsu), Mark Underwood (Krypton Brothers; Synchrony Financial), and Akhil Manchanda (GE) for the Security and Privacy Subgroup; David Boyd (InCadence Strategic Solutions), Orit Levin (Microsoft), Don Krapohl (Augmented Intelligence), and James Ketner (AT\&T) for the Reference Architecture Subgroup; and Russell Reinsch (Center for Government Interoperability), David Boyd (InCadence Strategic Solutions), Carl Buffington (Vistronix), and Dan McClary (Oracle), for the Standards Roadmap Subgroup.

The editors for this document were the following:

- Version 1: David Boyd (InCadence Strategic Solutions), Carl Buffington (Vistronix), and Wo Chang (NIST)

- Version 2: Russell Reinsch (Center for Government Interoperability) and Wo Chang (NIST)

Laurie Aldape (Energetics Incorporated) and Elizabeth Lennon (NIST) provided editorial assistance across all NBDIF volumes.

NIST SP1500-7, Version 2 has been collaboratively authored by the NBD-PWG. As of the date of this publication, there are over six hundred NBD-PWG participants from industry, academia, and government. Federal agency participants include the National Archives and Records Administration (NARA), National Aeronautics and Space Administration (NASA), National Science Foundation (NSF), and the U.S. Departments of Agriculture, Commerce, Defense, Energy, Census, Health and Human Services, Homeland Security, Transportation, Treasury, and Veterans Affairs.

NIST would like to acknowledge the specific contributions ${ }^{a}$ to this volume, during Version 1 and/or Version 2 activities, by the following NBD-PWG members:

\author{
Chaitan Baru \\ University of California, San \\ Diego, Supercomputer Center \\ David Boyd \\ InCadence Strategic Services \\ Carl Buffington \\ Vistronix \\ Wo Chang \\ NIST \\ Yuri Demchenko \\ University of Amsterdam \\ Kate Dolan \\ CTFC \\ Frank Farance \\ Farance, Inc.
}

Bruno Kelpsas
Microsoft Consultant
Pavithra Kenjige
PK Technologies
Brenda Kirkpatrick
Hewlett-Packard
Donald Krapohl
Augmented Intelligence
Luca Lepori
Data Hold
Orit Levin
Microsoft
Jan Levine
kloudtrack
Serge Mankovski

Shawn Miller
U.S. Department of Veterans
Affairs

William Miller MaCT USA

Sanjay Mishra
Verizon
Quyen Nguyen
NARA
Russell Reinsch
Center for Government
Interoperability

John Rogers

Hewlett-Packard

Doug Scrimager

\footnotetext{
a “Contributors” are members of the NIST Big Data Public Working Group who dedicated great effort to prepare and gave substantial time on a regular basis to research and development in support of this document.
} 
NIST BIG DATA INTEROPERABILITY FRAMEWORK: VOLUME 7, STANDARDS ROADMAP

Nancy Grady

SAIC

Keith Hare

JCC Consulting, Inc.

Zane Harvey
CA Technologies

Robert Marcus

ET-Strategies

Gary Mazzaferro

AlloyCloud, Inc.
Slalom Consulting

Cherry Tom

IEEE-SA

Mark Underwood

Krypton Brothers; Synchrony

Financial 


\section{TABLE OF CONTENTS}

EXECUTIVE SUMMARY

1 INTRODUCTION

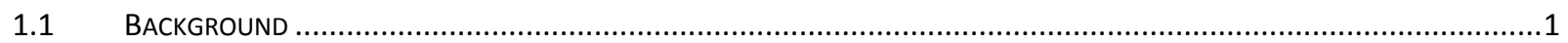

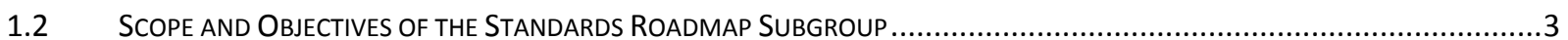

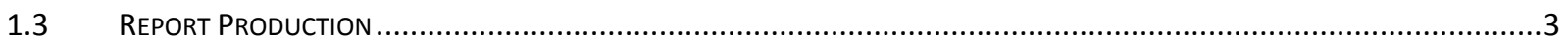

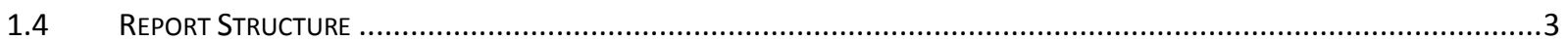

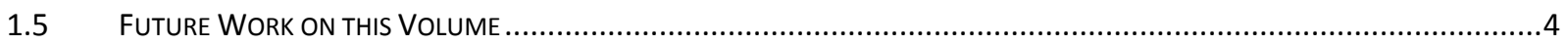

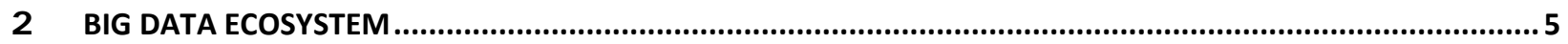

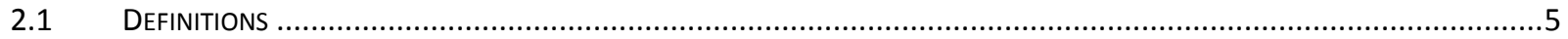

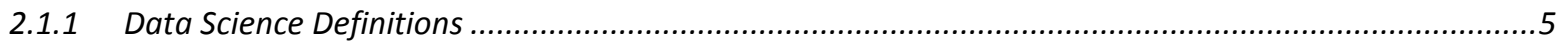

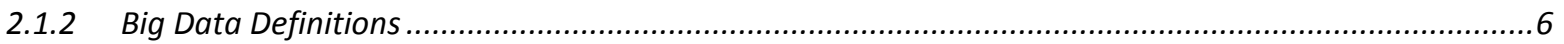

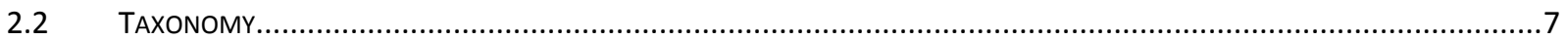

2.3 USE CASES

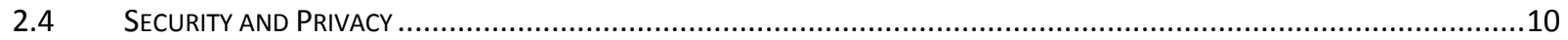

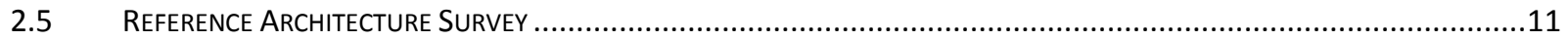

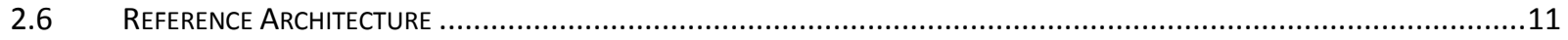

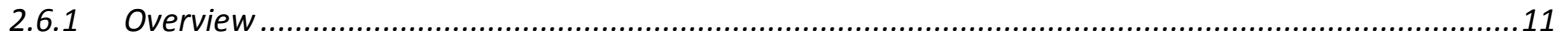

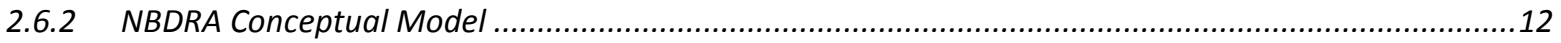

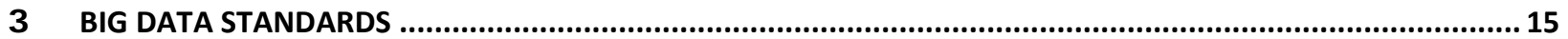

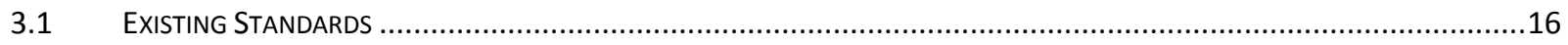

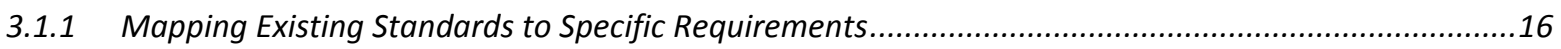

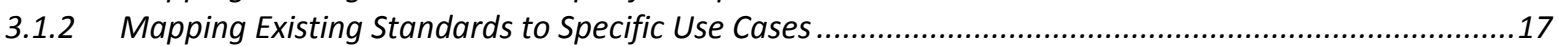

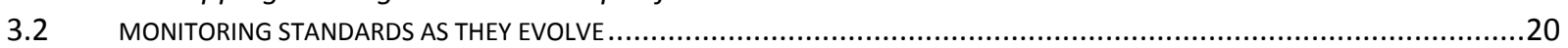

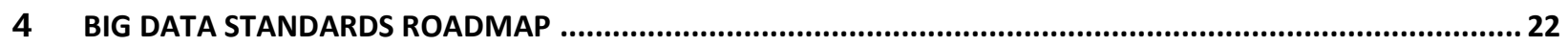

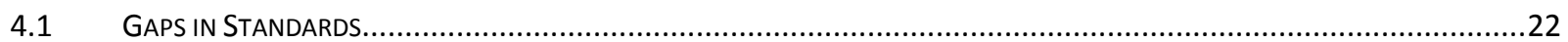

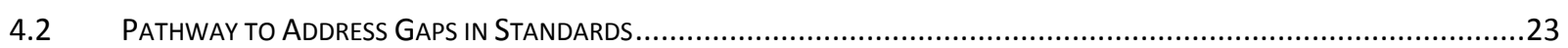

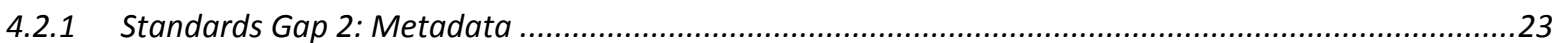

4.2.2 Standards Gap 4: Non-relational Database Query, Search and Information Retrieval ........................23

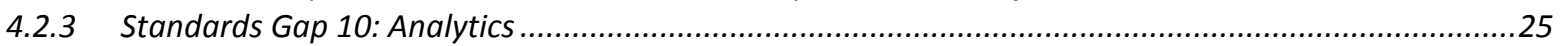

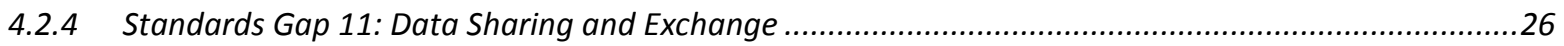

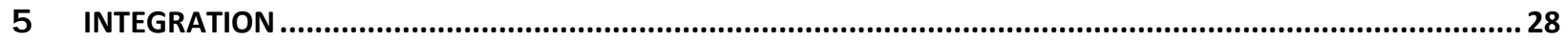

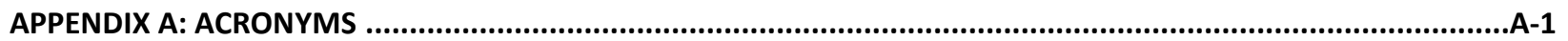

APPENDIX B: COLLECTION OF BIG DATA RELATED STANDARDS …..................................................................

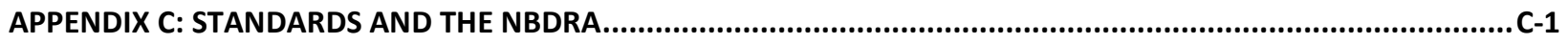

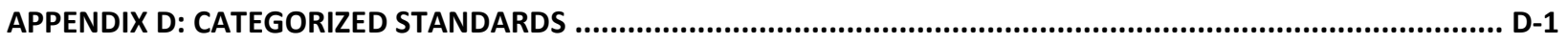

APPENDIX E: REFERENCES ..................................................................................................................................... E-1

\section{LIST OF FI GURES}

Figure 1: NIST Big DAtA Reference ARChItECture TAXonomy. 8 
NIST Big DATA INTEROPERABILITY FRAMEWORK: VOLUME 7, STANDARDS ROADMAP

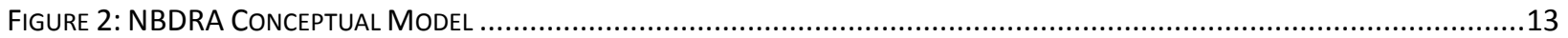

\section{LIST OF TABLES}

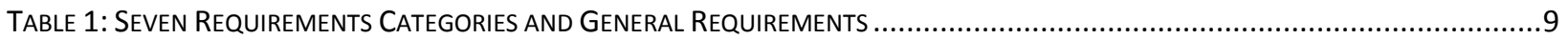

Table 2: Mapping USe CaSe Characterization Categories to Reference ArChitecture Components and FabricS................12

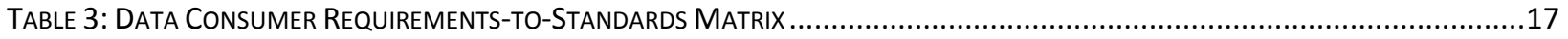

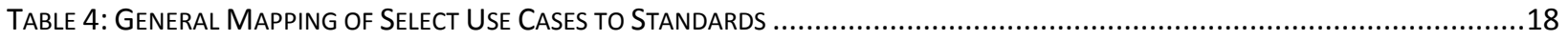

TABLE 5: EXCERPT FROM USE CASE DOCUMENT M0165－DETAILED MAPPING TO STANDARDS............................................18

TABLE 6: EXCERPT FROM USE CASE DOCUMENT M0215-DETAILED MAPPING TO STANDARDS...........................................19

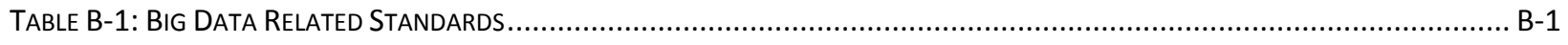

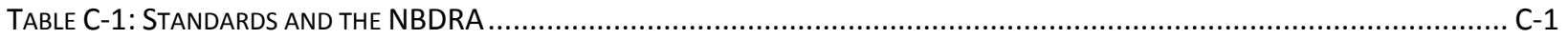

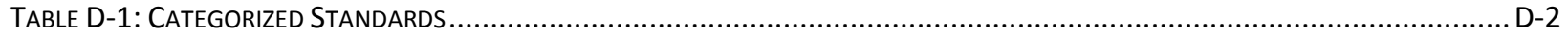




\section{EXECUTIVE SUMMARY}

To provide a common Big Data framework, the NIST Big Data Public Working Group (NBD-PWG) is creating vendor-neutral, technology- and infrastructure-agnostic deliverables, which include the development of consensus-based definitions, taxonomies, a reference architecture, and a roadmap. This document, NIST Big Data Interoperability Framework (NBDIF): Volume 7, Standards Roadmap, summarizes the work of the other NBDPWG subgroups (presented in detail in the other volumes of this series) and presents the work of the NBD-PWG Standards Roadmap Subgroup. The NBD-PWG Standards Roadmap Subgroup investigated existing standards that relate to Big Data, initiated a mapping effort to connect existing standards with both Big Data requirements and use cases (developed by the Use Cases and Requirements Subgroup), and explored gaps in the Big Data standards.

The NBDIF consists of nine volumes, each of which addresses a specific key topic, resulting from the work of the NBD-PWG. The nine NBDIF volumes, which can be downloaded from https://bigdatawg.nist.gov/V2_output_docs.php, are as follows:

- Volume 1, Definitions [1]

- Volume 2, Taxonomies [2]

- Volume 3, Use Cases and General Requirements [3]

- Volume 4, Security and Privacy [4]

- Volume 5, Architectures White Paper Survey [5]

- Volume 6, Reference Architecture [6]

- Volume 7, Standards Roadmap (this volume)

- Volume 8, Reference Architecture Interfaces [7]

- Volume 9, Adoption and Modernization [8]

The NBDIF will be released in three versions, which correspond to the three development stages of the NBDPWG work. The three stages aim to achieve the following with respect to the NIST Big Data Reference Architecture (NBDRA).

Stage 1: Identify the high-level Big Data reference architecture key components, which are technology-, infrastructure-, and vendor-agnostic;

Stage 2: Define general interfaces between the NBDRA components; and

Stage 3: Validate the NBDRA by building Big Data general applications through the general interfaces.

Potential areas of future work for the Subgroup during Stage 3 are highlighted in Section 1.5 of each volume. The current effort documented in this volume reflects concepts developed within the rapidly evolving field of Big Data. 


\section{INTRODUCTION}

\subsection{BACKGROUND}

There is broad agreement among commercial, academic, and government leaders about the remarkable potential of Big Data to spark innovation, fuel commerce, and drive progress. Big Data is the common term used to describe the deluge of data in today's networked, digitized, sensor-laden, and informationdriven world. The availability of vast data resources carries the potential to answer questions previously out of reach, including the following:

- How can a potential pandemic reliably be detected early enough to intervene?

- Can new materials with advanced properties be predicted before these materials have ever been synthesized?

- How can the current advantage of the attacker over the defender in guarding against cybersecurity threats be reversed?

There is also broad agreement on the ability of Big Data to overwhelm traditional approaches. The growth rates for data volumes, speeds, and complexity are outpacing scientific and technological advances in data analytics, management, transport, and data user spheres.

Despite widespread agreement on the inherent opportunities and current limitations of Big Data, a lack of consensus on some important fundamental questions continues to confuse potential users and stymie progress. These questions include the following:

- How is Big Data defined?

- What attributes define Big Data solutions?

- What is new in Big Data?

- What is the difference between Big Data and bigger data that has been collected for years?

- How is Big Data different from traditional data environments and related applications?

- What are the essential characteristics of Big Data environments?

- How do these environments integrate with currently deployed architectures?

- What are the central scientific, technological, and standardization challenges that need to be addressed to accelerate the deployment of robust, secure Big Data solutions?

Within this context, on March 29, 2012, the White House announced the Big Data Research and Development Initiative. The initiative's goals include helping to accelerate the pace of discovery in science and engineering, strengthening national security, and transforming teaching and learning by improving analysts' ability to extract knowledge and insights from large and complex collections of digital data.

Six federal departments and their agencies announced more than \$200 million in commitments spread across more than 80 projects, which aim to significantly improve the tools and techniques needed to access, organize, and draw conclusions from huge volumes of digital data. The initiative also challenged industry, research universities, and nonprofits to join with the federal government to make the most of the opportunities created by Big Data.

Motivated by the White House initiative and public suggestions, the National Institute of Standards and Technology (NIST) accepted the challenge to stimulate collaboration among industry professionals to further the secure and effective adoption of Big Data. As one result of NIST's Cloud and Big Data Forum held on January 15-17, 2013, there was strong encouragement for NIST to create a public working group 
for the development of a Big Data Standards Roadmap. Forum participants noted that this roadmap should define and prioritize Big Data requirements, including interoperability, portability, reusability, extensibility, data usage, analytics, and technology infrastructure. In doing so, the roadmap would accelerate the adoption of the most secure and effective Big Data techniques and technology.

On June 19, 2013, the NIST Big Data Public Working Group (NBD-PWG) was launched with extensive participation by industry, academia, and government from across the nation. The scope of the NBD-PWG involves forming a community of interests from all sectors-including industry, academia, and government - with the goal of developing consensus on definitions, taxonomies, secure reference architectures, security and privacy, and, from these, a standards roadmap. Such a consensus would create a vendor-neutral, technology- and infrastructure-independent framework that would enable Big Data stakeholders to identify and use the best analytics tools for their processing and visualization requirements on the most suitable computing platform and cluster, while also allowing added value from Big Data service providers.

The NIST Big Data Interoperability Framework (NBDIF) will be released in three versions, which correspond to the three stages of the NBD-PWG work. The three stages aim to achieve the following with respect to the NIST Big Data Reference Architecture (NBDRA).

Stage 1: Identify the high-level Big Data reference architecture key components, which are technology, infrastructure, and vendor agnostic;

Stage 2: Define general interfaces between the NBDRA components; and

Stage 3: Validate the NBDRA by building Big Data general applications through the general interfaces.

On September 16, 2015, seven NBDIF Version 1 volumes were published

(http://bigdatawg.nist.gov/V1_output_docs.php), each of which addresses a specific key topic, resulting from the work of the NBD-PWG. The seven volumes are as follows:

- Volume 1, Definitions [1]

- Volume 2, Taxonomies [2]

- Volume 3, Use Cases and General Requirements [3]

- Volume 4, Security and Privacy [4]

- Volume 5, Architectures White Paper Survey [5]

- Volume 6, Reference Architecture [6]

- Volume 7, Standards Roadmap (this volume)

Currently, the NBD-PWG is working on Stage 2 with the goals to enhance the Version 1 content, define general interfaces between the NBDRA components by aggregating low-level interactions into high-level general interfaces, and demonstrate how the NBDRA can be used. As a result of the Stage 2 work, the following two additional NBDIF volumes have been developed.

- Volume 8, Reference Architecture Interfaces [7]

- Volume 9, Adoption and Modernization [8]

Version 2 of the NBDIF volumes, resulting from Stage 2 work, can be downloaded from the NBD-PWG website (https://bigdatawg.nist.gov/V2_output_docs.php). Potential areas of future work for each volume during Stage 3 are highlighted in Section 1.5 of each volume. The current effort documented in this volume reflects concepts developed within the rapidly evolving field of Big Data. 


\subsection{SCOPE AND OBJECTIVES OF THE STANDARDS ROADMAP SUBGROUP}

The NBD-PWG Standards Roadmap Subgroup focused on forming a community of interest from industry, academia, and government, with the goal of developing a standards roadmap. The Subgroup's approach included the following:

- Collaborate with the other four NBD-PWG subgroups;

- Review products of the other four subgroups including taxonomies, use cases, general requirements, and reference architecture;

- Gain an understanding of what standards are available or under development that may apply to Big Data;

- Perform standards, gap analysis and document the findings;

- Document vision and recommendations for future standards activities;

- Identify possible barriers that may delay or prevent adoption of Big Data; and

- Identify a few areas where new standards could have a significant impact.

The goals of the Subgroup will be realized throughout the three planned phases of the NBD-PWG work, as outlined in Section 1.1.

Within the multitude of standards applicable to data and information technology, the Subgroup focused on standards that: (1) apply to situations encountered in Big Data; (2) facilitate interfaces between NBDRA components (difference between Implementer [encoder] or User [decoder] may be nonexistent), (3) facilitate handling characteristics, and (4) represent a fundamental function.

\subsection{REPORT PRODUCTION}

The NBDIF: Volume 7, Standards Roadmap is one of nine volumes, whose overall aims are to define and prioritize Big Data requirements, including interoperability, portability, reusability, extensibility, data usage, analytic techniques, and technology infrastructure to support secure and effective adoption of Big Data. The NBDIF: Volume 7, Standards Roadmap is dedicated to developing a consensus vision with recommendations on how Big Data should move forward specifically in the area of standardization. In the first phase, the Subgroup focused on the identification of existing standards relating to Big Data and inspection of gaps in those standards. During the second phase, the Subgroup mapped standards to requirements identified by the NBD-PWG, mapped standards to use cases gathered by the NBD-PWG, and discussed possible pathways to address gaps in the standards.

To achieve technical and high-quality document content, this document will go through a public comments period along with NIST internal review.

\subsection{REPORT STRUCTURE}

Following the introductory material presented in Section 1, the remainder of this document is organized as follows:

- Section 2 summarizes the work developed by the other four subgroups and presents the mapping of standards to requirements and standards to use cases.

- $\quad$ Section 3 reviews existing standards that may apply to Big Data, provides two different viewpoints for understanding the standards landscape, and considers the maturation of standards.

- Section 4 presents current gaps in Big Data standards, discusses possible pathways to address the gaps, and examines areas where the development of standards could have significant impact. 


\subsection{FUTURE WORK ON THIS VOLUME}

The NBDIF will be released in three versions, which correspond to the three stages of the NBD-PWG work, as outlined in Section 1.1. Version 3 activities may focus on the following:

- Document recommendations for future standards activities.

- Further map standards to NBDRA components and the interfaces between them.

- Map additional requirements to standards.

- Map additional use cases to standards.

- Explore the divergence of technologies and common project methodologies and the impact on standards creation.

- Investigate the impact of standards for IoT, including a recognized need in the area of encrypted network traffic.

- Consider the need for standards in the areas of network connectivity, complex event processing, platform as a service (PaaS), and crowdsourced mediation.

- Explore existing and gaps in data standards, including topics such as types of datasets, application-level services, open data, and government initiatives.

- Consider commercial datasets and open marketplaces.

- Construct gap closure strategies.

- Map standards to additional use cases (e.g., use cases 2, 6, 34). 


\section{BIG DATA ECOSYSTEM}

The exponential growth of data is already resulting in the development of new theories addressing topics from synchronization of data across large distributed computing environments to addressing consistency in high-volume and high-velocity environments. As actual implementations of technologies are proven, reference implementations will evolve based on community accepted open source efforts.

The NBDIF is intended to represent the overall topic of Big Data, grouping the various aspects of the topic into high-level facets of the ecosystem. At the forefront of the construct, the NBD-PWG laid the groundwork for construction of a reference architecture. Development of a Big Data reference architecture involves a thorough understanding of current techniques, issues, concerns, and other topics. To this end, the NBD-PWG collected use cases to gain an understanding of current applications of Big Data, conducted a survey of reference architectures to understand commonalities within Big Data architectures in use, developed a taxonomy to understand and organize the information collected, and reviewed existing Big Data-relevant technologies and trends.

From the collected use cases and architecture survey information ${ }^{\mathrm{b}}$, the NBD-PWG created the NBDRA, which is a high-level conceptual model designed to serve as a tool to facilitate open discussion of the requirements, structures, and operations inherent in Big Data. These NBD-PWG activities and functional components were used as input during the development of the entire NIST Big Data Interoperability Framework.

The remainder of Section 2 summarizes the NBD-PWG work contained in other NBDIF Volumes.

\subsection{DEFINITIONS}

There are two fundamental concepts in the emerging discipline of Big Data that have been used to represent multiple concepts. These two concepts, Big Data and Data Science, are broken down into individual terms and concepts in the following subsections. As a basis for discussions of the NBDRA and related standards, associated terminology is defined in subsequent subsections. The NBDIF: Volume 1, Definitions explores additional concepts and terminology surrounding Big Data.

\subsubsection{DATA SCIENCE DEFINITIONS}

In its purest form, data science is the fourth paradigm of science, following theory, experiment, and computational science. The fourth paradigm is a term coined by Dr. Jim Gray in 2007 to refer to the conduct of data analysis as an empirical science, learning directly from data itself. Data science as a paradigm would refer to the formulation of a hypothesis, the collection of the data-new or preexistingto address the hypothesis, and the analytical confirmation or denial of the hypothesis (or the determination that additional information or study is needed.) As in any experimental science, the result could in fact be that the original hypothesis itself needs to be reformulated. The key concept is that data science is an empirical science, performing the scientific process directly on the data. Note that the hypothesis may be driven by a business need, or can be the restatement of a business need in terms of a technical hypothesis.

Data science is the extraction of useful knowledge directly from data through a process of discovery, or of hypothesis formulation and hypothesis testing.

\footnotetext{
${ }^{\mathrm{b}}$ See NBDIF: Volumes 3, 5, and 6, version 1 for additional information on the use cases, reference architecture information collection, and development of the NBDRA.
} 
While the above definition of the data science paradigm refers to learning directly from data, in the Big Data paradigm, this learning must now implicitly involve all steps in the data life cycle, with analytics being only a subset. Data science can be understood as the activities happening in the data layer of the system architecture to extract knowledge from the raw data.

The data life cycle is the set of processes that transform raw data into actionable knowledge, which includes data collection, preparation, analytics, visualization, and access.

Traditionally, the term analytics has been used as one of the steps in the data life cycle of collection, preparation, analysis, and action.

Analytics is the synthesis of knowledge from information.

\subsubsection{BIG DATA DEFINITIONS}

Big Data refers to the inability of traditional data architectures to efficiently handle the new datasets. Characteristics of Big Data that force new architectures are volume (i.e., the size of the dataset) and variety (i.e., data from multiple repositories, domains, or types), and the data in motion characteristics of velocity (i.e., rate of flow) and variability (i.e., the change in other characteristics). These characteristics - volume, variety, velocity, and variability — are known colloquially as the Vs of Big Data and are further discussed in the NBDIF: Volume 1, Definitions.

Each of these characteristics influences the overall design of a Big Data system, resulting in different data system architectures or different data life cycle process orderings to achieve needed efficiencies. A number of other terms are also used, several of which refer to the analytics process instead of new Big Data characteristics. The following Big Data definitions have been used throughout the seven volumes of the NBDIF and are fully described in the NBDIF: Volume 1, Definitions.

Big Data consists of extensive datasets-primarily in the characteristics of volume, variety, velocity, and/or variability — that require a scalable architecture for efficient storage, manipulation, and analysis.

The Big Data paradigm consists of the distribution of data systems across horizontally coupled, independent resources to achieve the scalability needed for the efficient processing of extensive datasets.

Veracity refers to accuracy of the data.

Value refers to the inherent wealth, economic and social, embedded in any dataset.

Volatility refers to the tendency for data structures to change over time.

Validity refers to appropriateness of the data for its intended use

Like many terms that have come into common usage in the current information age, Big Data has many possible meanings depending on the context from which it is viewed. Big Data discussions are complicated by the lack of accepted definitions, taxonomies, and common reference views. The products of the NBD-PWG are designed to specifically address the lack of consistency. The NBD-PWG is aware that both technical and nontechnical audiences need to keep abreast of the rapid changes in the Big Data landscape as those changes can affect their ability to manage information in effective ways.

For each of these two unique audiences, the consumption of written, audio, or video information on Big Data is reliant on certain accepted definitions for terms. For nontechnical audiences, a method of expressing the Big Data aspects in terms of volume, variety and velocity, known as the Vs, became popular for its ability to frame the somewhat complex concepts of Big Data in simpler, more digestible ways. 
Similar to the who, what, and where interrogatives used in journalism, the Vs represent checkboxes for listing the main elements required for narrative storytelling about Big Data. While not precise from a terminology standpoint, they do serve to motivate discussions that can be analyzed more closely in other settings such as those involving technical audiences requiring language which more closely corresponds to the complete corpus of terminology used in the field of study.

Tested against the corpus of use, a definition of Big Data can be constructed by considering the essential technical characteristics in the field of study. These characteristics tend to cluster into the following five distinct segments:

1. Irregular or heterogeneous data structures, their navigation, query, and data-typing (i.e., variety);

2. The need for computation and storage parallelism and its management during processing of large datasets (i.e., volume);

3. Descriptive data and self-inquiry about objects for real-time decision making (i.e., validity/veracity);

4. The rate of arrival of the data (i.e., velocity); and

5. Presentation and aggregation of such datasets (i.e., visualization). [9]

With respect to computation parallelism, issues concern the unit of processing (e.g., thread, statement, block, process, and node), contention methods for shared access, and begin-suspend-resume-completiontermination processing.

Descriptive data is also known as metadata. Self-inquiry is often referred to as reflection or introspection in some programming paradigms.

With respect to visualization, visual limitations concern how much information a human can usefully process on a single display screen or sheet of paper. For example, the presentation of a connection graph of 500 nodes might require more than 20 rows and columns, along with the connections or relationships among each of the pairs. Typically, this is too much for a human to comprehend in a useful way. Big Data presentation concerns itself with reformulating the information in a way that makes the data easier for humans to consume.

It is also important to note that Big Data is not necessarily about a large amount of data because many of these concerns can arise when dealing with smaller, less than gigabyte datasets. Big Data concerns typically arise in processing large amounts of data because some or all of the four main characteristics (irregularity, parallelism, real-time metadata, presentation / visualization) are unavoidable in such large datasets.

\subsection{TAXONOMY}

The NBD-PWG Definitions and Taxonomy Subgroup developed a hierarchy of reference architecture components. Additional taxonomy details are presented in the NBDIF: Volume 2, Taxonomy.

Figure 1 outlines potential actors for the seven roles developed by the NBD-PWG Definition and Taxonomy Subgroup. The dark blue boxes contain the name of the role at the top with potential actors listed directly below. 


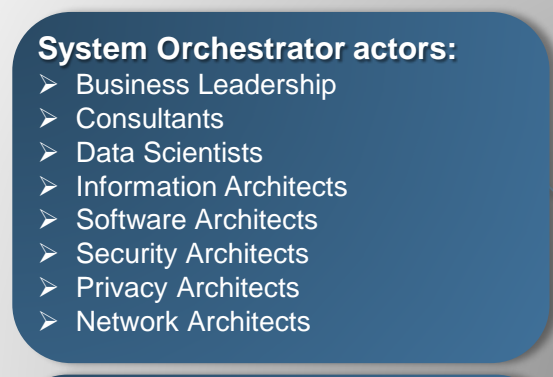

\section{Data Provider actors:}

$>$ Enterprises

$>$ Public Agencies

$>$ Researchers and Scientists

$>$ Search Engines

$>$ Web, FTP and Other Applications

$>$ Network Operators

End Users

\section{Security and Privacy Fabric \\ actors: \\ $>$ Corporate Security Officer \\ $>$ Security Specialist}

Big Data Application Provider actors:

$>$ Application Specialists

$>$ Platform Specialists

$>$ Consultants

Big Data Framework Provider actors:

$>$ In-house Clusters

$>$ Data Centers

$>$ Cloud Providers

Reference Architecture Taxonomy
Data Consumer actors:

$>$ End Users

$>$ Researchers

$>$ Applications

Systems

Management Fabric actors:

$>$ In-house Staff

$>$ Data Center Management

$>$ Cloud Providers

Figure 1: NIST Big Data Reference Architecture Taxonomy

\subsection{USE CASES}

A consensus list of Big Data requirements across stakeholders was developed by the NBD-PWG Use Cases and Requirements Subgroup. The development of requirements included gathering and understanding various use cases from the nine diversified areas, or application domains, listed below.

- Government Operation;

- Commercial;

- Defense;

- Healthcare and Life Sciences;

- Deep Learning and Social Media;

- The Ecosystem for Research;

- Astronomy and Physics;

- Earth, Environmental, and Polar Science; and

- Energy.

Participants in the NBD-PWG Use Cases and Requirements Subgroup and other interested parties supplied publicly available information for various Big Data architecture examples from the nine application domains, which developed organically from the 51 use cases collected by the Subgroup.

After collection, processing, and review of the use cases, requirements within seven Big Data characteristic categories were extracted from the individual use cases. Requirements are the challenges limiting further use of Big Data. The complete list of requirements extracted from the use cases is presented in the document NBDIF: Volume 3, Use Cases and General Requirements.

The use case specific requirements were then aggregated to produce high-level general requirements, within seven characteristic categories. The seven categories are as follows: 
- Data source requirements (relating to data size, format, rate of growth, at rest, etc.);

- Data transformation provider (i.e., data fusion, analytics);

- Capabilities provider (i.e., software tools, platform tools, hardware resources such as storage and networking);

- Data consumer (i.e., processed results in text, table, visual, and other formats);

- Security and privacy;

- Life cycle management (i.e., curation, conversion, quality check, pre-analytic processing); and

- Other requirements.

The general requirements, created to be vendor-neutral and technology-agnostic, are organized into seven categories in Table 1 below.

\section{Table 1: Seven Requirements Categories and General Requirements}

\begin{tabular}{|c|c|}
\hline \multicolumn{2}{|c|}{ DATA SOURCE REQUIREMENTS (DSR) } \\
\hline DSR-1 & $\begin{array}{l}\text { Needs to support reliable real-time, asynchronous, streaming, and batch processing to collect data from } \\
\text { centralized, distributed, and cloud data sources, sensors, or instruments. }\end{array}$ \\
\hline DSR-2 & $\begin{array}{l}\text { Needs to support slow, bursty, and high-throughput data transmission between data sources and } \\
\text { computing clusters. }\end{array}$ \\
\hline DSR-3 & $\begin{array}{l}\text { Needs to support diversified data content ranging from structured and unstructured text, document, } \\
\text { graph, web, geospatial, compressed, timed, spatial, multimedia, simulation, and instrumental data. }\end{array}$ \\
\hline \multicolumn{2}{|c|}{ TRANSFORMATION PROVIDER REQUIREMENTS (TPR) } \\
\hline TPR-1 & Needs to support diversified compute-intensive, analytic processing, and machine learning techniques. \\
\hline TPR-2 & Needs to support batch and real-time analytic processing. \\
\hline TPR-3 & Needs to support processing large diversified data content and modeling. \\
\hline TPR-4 & Needs to support processing data in motion (e.g., streaming, fetching new content, tracking). \\
\hline \multicolumn{2}{|c|}{ CAPABILITY PROVIDER REQUIREMENTS (CPR) } \\
\hline CPR-1 & Needs to support legacy and advanced software packages (software). \\
\hline CPR-2 & Needs to support legacy and advanced computing platforms (platform). \\
\hline CPR-3 & $\begin{array}{l}\text { Needs to support legacy and advanced distributed computing clusters, co-processors, input output } \\
\text { processing (infrastructure). }\end{array}$ \\
\hline CPR-4 & Needs to support elastic data transmission (networking). \\
\hline CPR-5 & Needs to support legacy, large, and advanced distributed data storage (storage). \\
\hline CPR-6 & $\begin{array}{l}\text { Needs to support legacy and advanced executable programming: applications, tools, utilities, and } \\
\text { libraries (software). }\end{array}$ \\
\hline \multicolumn{2}{|c|}{ DATA CONSUMER REQUIREMENTS (DCR) } \\
\hline DCR-1 & $\begin{array}{l}\text { Needs to support fast searches ( } \sim 0.1 \text { seconds) from processed data with high relevancy, accuracy, and } \\
\text { recall. }\end{array}$ \\
\hline DCR-2 & Needs to support diversified output file formats for visualization, rendering, and reporting. \\
\hline DCR-3 & Needs to support visual layout for results presentation. \\
\hline DCR-4 & Needs to support rich user interface for access using browser, visualization tools. \\
\hline DCR-5 & Needs to support high-resolution, multidimensional layer of data visualization. \\
\hline DCR-6 & Needs to support streaming results to clients. \\
\hline \multicolumn{2}{|c|}{ SECURITY AND PRIVACY REQUIREMENTS (SPR) } \\
\hline SPR-1 & Needs to protect and preserve security and privacy of sensitive data. \\
\hline SPR-2 & $\begin{array}{l}\text { Needs to support sandbox, access control, and multilevel, policy-driven authentication on protected } \\
\text { data. }\end{array}$ \\
\hline
\end{tabular}




\begin{tabular}{|l|l|}
\hline \multicolumn{2}{|l|}{ LIFE CYCLE MANAGEMENT REQUIREMENTS (LMR) } \\
\hline LMR-1 & $\begin{array}{l}\text { Needs to support data quality curation including preprocessing, data clustering, classification, reduction, } \\
\text { and format transformation. }\end{array}$ \\
\hline LMR-2 & Needs to support dynamic updates on data, user profiles, and links. \\
\hline LMR-3 & Needs to support data life cycle and long-term preservation policy, including data provenance. \\
\hline LMR-4 & Needs to support data validation. \\
\hline LMR-5 & Needs to support human annotation for data validation. \\
\hline LMR-6 & Needs to support prevention of data loss or corruption. \\
\hline LMR-7 & Needs to support multisite archives. \\
\hline LMR-8 & Needs to support persistent identifier and data traceability. \\
\hline LMR-9 & Needs to support standardizing, aggregating, and normalizing data from disparate sources. \\
\hline OTHER REQUIREMENTS (OR) \\
\hline OR-1 & Needs to support rich user interface from mobile platforms to access processed results. \\
\hline OR-2 & Needs to support performance monitoring on analytic processing from mobile platforms. \\
\hline OR-3 & Needs to support rich visual content search and rendering from mobile platforms. \\
\hline OR-4 & Needs to support mobile device data acquisition. \\
\hline OR-5 & Needs to support security across mobile devices. \\
\hline
\end{tabular}

Additional information about the Use Cases and Requirements Subgroup, use case collection, analysis of the use cases, and generation of the use case requirements are presented in the NBDIF: Volume 3, Use Cases and General Requirements document.

\subsection{SECURITY AND PRIVACY}

Security and privacy measures for Big Data involve a different approach than traditional systems. Big Data is increasingly stored on public cloud infrastructure built by various hardware, operating systems, and analytical software. Traditional security approaches usually addressed small-scale systems holding static data on firewalled and semi-isolated networks. The surge in streaming cloud technology necessitates extremely rapid responses to security issues and threats. [10]

Security and privacy considerations are a fundamental aspect of Big Data and affect all components of the NBDRA. This comprehensive influence is depicted in Figure 2 by the grey rectangle marked "Security and Privacy" surrounding all the reference architecture components. At a minimum, a Big Data reference architecture will provide verifiable compliance with both governance, risk management, and compliance (GRC) and confidentiality, integrity, and availability (CIA) policies, standards, and best practices. Additional information on the processes and outcomes of the NBD PWG Security and Privacy Subgroup are presented in NBDIF: Volume 4, Security and Privacy.

The NBD-PWG Security and Privacy Subgroup began this effort by identifying ways that security and Privacy in Big Data projects can be different from traditional implementations. While not all concepts apply all the time, the following seven observations were considered representative of a larger set of differences:

1. Big Data projects often encompass heterogeneous components in which a single security scheme has not been designed from the outset.

2. Most security and privacy methods have been designed for batch or online transaction processing systems. Big Data projects increasingly involve one or more streamed data sources that are used in conjunction with data at rest, creating unique security and privacy scenarios. 
3. The use of multiple Big Data sources not originally intended to be used together can compromise privacy, security, or both. Approaches to de-identify personally identifiable information (PII) that were satisfactory prior to Big Data may no longer be adequate, while alternative approaches to protecting privacy are made feasible. Although de-identification techniques can apply to data from single sources as well, the prospect of unanticipated multiple datasets exacerbates the risk of compromising privacy.

4. An increased reliance on sensor streams, such as those anticipated with the Internet of Things (IoT; e.g., smart medical devices, smart cities, smart homes) can create vulnerabilities that were more easily managed before amassed to Big Data scale.

5. Certain types of data thought to be too big for analysis, such as geospatial and video imaging, will become commodity Big Data sources. These uses were not anticipated and/or may not have implemented security and privacy measures.

6. Issues of veracity, context, provenance, and jurisdiction are greatly magnified in Big Data. Multiple organizations, stakeholders, legal entities, governments, and an increasing number of citizens will find data about themselves included in Big Data analytics.

7. Volatility is significant because Big Data scenarios envision that data is permanent by default. Security is a fast-moving field with multiple attack vectors and countermeasures. Data may be preserved beyond the lifetime of the security measures designed to protect it.

8. Data and code can more readily be shared across organizations, but many standards presume management practices that are managed inside a single organizational framework.

\subsection{REFERENCE ARCHITECTURE SURVEY}

The NBD-PWG Reference Architecture Subgroup conducted the reference architecture survey to advance understanding of the operational intricacies in Big Data and to serve as a tool for developing systemspecific architectures using a common reference framework. The Subgroup surveyed currently published Big Data platforms by leading companies or individuals supporting the Big Data framework and analyzed the collected material. This effort revealed a remarkable consistency between Big Data architectures. Survey details, methodology, and conclusions are reported in NBDIF: Volume 5, Architectures White Paper Survey.

\subsection{REFERENCE ARCHITECTURE}

\subsubsection{OVERVIEW}

The goal of the NBD-PWG Reference Architecture Subgroup is to develop a Big Data open reference architecture that facilitates the understanding of the operational intricacies in Big Data. It does not represent the system architecture of a specific Big Data system, but rather is a tool for describing, discussing, and developing system-specific architectures using a common framework of reference. The reference architecture achieves this by providing a generic high-level conceptual model that is an effective tool for discussing the requirements, structures, and operations inherent to Big Data. The model is not tied to any specific vendor products, services, or reference implementation, nor does it define prescriptive solutions that inhibit innovation.

The design of the NBDRA does not address the following:

- Detailed specifications for any organization's operational systems;

- Detailed specifications of information exchanges or services; and

- Recommendations or standards for integration of infrastructure products.

Building on the work from other subgroups, the NBD-PWG Reference Architecture Subgroup evaluated the general requirements formed from the use cases, evaluated the Big Data Taxonomy, performed a 
reference architecture survey, and developed the NBDRA conceptual model. The NBDIF: Volume 3, Use Cases and General Requirements document contains details of the Subgroup's work.

The use case characterization categories (from NBDIF: Volume 3, Use Cases and General Requirements) are listed below on the left and were used as input in the development of the NBDRA. Some use case characterization categories were renamed for use in the NBDRA. Table 2 maps the earlier use case terms directly to NBDRA components and fabrics.

\section{Table 2: Mapping Use Case Characterization Categories to Reference Architecture Components and Fabrics}

\begin{tabular}{|lll|}
\hline USE CASE CHARACTERIZATION & & $\begin{array}{l}\text { REFERENCE ARCHITECTURE COMPONENTS } \\
\text { AND FABRICS }\end{array}$ \\
\hline Data sources & $\rightarrow$ & Data Provider \\
\hline Data transformation & $\rightarrow$ & Big Data Application Provider \\
\hline Capabilities & $\rightarrow$ & Big Data Framework Provider \\
\hline Data consumer & $\rightarrow$ & Data Consumer \\
\hline Security and privacy & $\rightarrow$ & Security and Privacy Fabric \\
\hline Life cycle management & $\rightarrow$ & System Orchestrator; Management Fabric \\
\hline Other requirements & $\rightarrow$ & To all components and fabrics \\
\hline
\end{tabular}

\subsubsection{NBDRA CONCEPTUAL MODEL}

As discussed in Section 2, the NBD-PWG Reference Architecture Subgroup used a variety of inputs from other NBD-PWG subgroups in developing a vendor-neutral, technology- and infrastructure-agnostic conceptual model of Big Data architecture. This conceptual model, the NBDRA, is shown in Figure 2 and represents a Big Data system composed of five logical functional components connected by interoperability interfaces (i.e., services). Two fabrics envelop the components, representing the interwoven nature of management and security and privacy with all five of the components.

The NBDRA is intended to enable system engineers, data scientists, software developers, data architects, and senior decision makers to develop solutions to issues that require diverse approaches due to convergence of Big Data characteristics within an interoperable Big Data ecosystem. It provides a framework to support a variety of business environments, including tightly integrated enterprise systems and loosely coupled vertical industries, by enhancing understanding of how Big Data complements and differs from existing analytics, business intelligence, databases, and systems. 


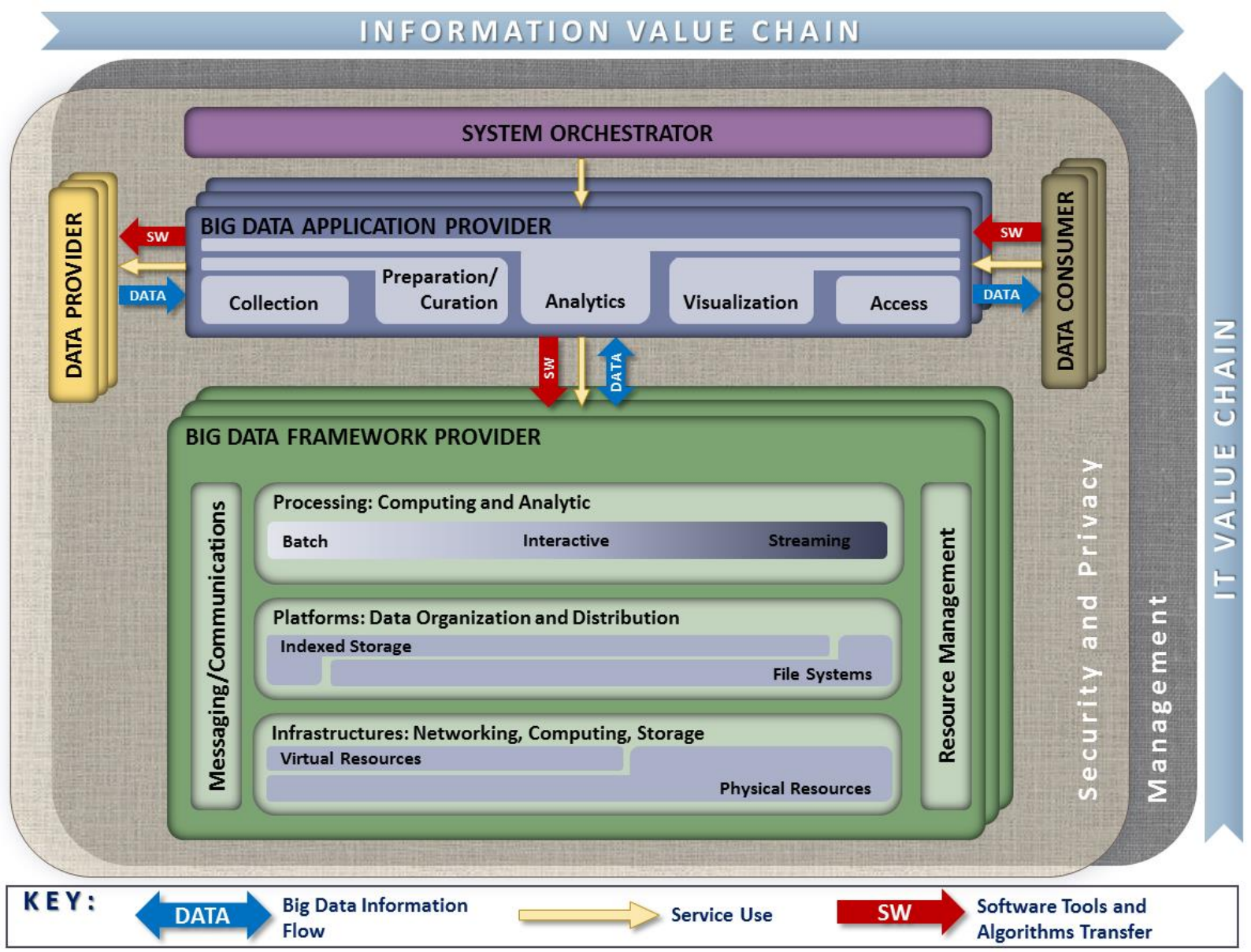

Figure 2: NBDRA Conceptual Model

Note: None of the terminology or diagrams in these documents is intended to be normative or to imply any business or deployment model. The terms provider and consumer as used are descriptive of general roles and are meant to be informative in nature.

The NBDRA is organized around five major roles and multiple sub-roles aligned along two axes representing the two Big Data value chains: Information Value (horizontal axis) and Information Technology (IT; vertical axis). Along the information axis, the value is created by data collection, integration, analysis, and applying the results following the value chain. Along the IT axis, the value is created by providing networking, infrastructure, platforms, application tools, and other IT services for hosting of and operating the Big Data in support of required data applications. At the intersection of both axes is the Big Data Application Provider role, indicating that data analytics and its implementation provide the value to Big Data stakeholders in both value chains.

The five main NBDRA roles, shown in Figure 2 and discussed in detail in Section 3, represent different technical roles that exist in every Big Data system. These roles are the following:

- System Orchestrator,

- Data Provider,

- Big Data Application Provider,

- Big Data Framework Provider, and

- Data Consumer. 
The two fabric roles shown in Figure 2 encompassing the five main roles are:

- Management, and

- Security and Privacy.

These two fabrics provide services and functionality to the five main roles in the areas specific to Big Data and are crucial to any Big Data solution.

The DATA arrows in Figure 2 show the flow of data between the system's main roles. Data flows between the roles either physically (i.e., by value) or by providing its location and the means to access it (i.e., by reference). The $\mathbf{S W}$ arrows show transfer of software tools for processing of Big Data in situ. The Service Use arrows represent software programmable interfaces. While the main focus of the NBDRA is to represent the run-time environment, all three types of communications or transactions can happen in the configuration phase as well. Manual agreements (e.g., service-level agreements) and human interactions that may exist throughout the system are not shown in the NBDRA.

The roles in the Big Data ecosystem perform activities and are implemented via functional components. In system development, actors and roles have the same relationship as in the movies, but system development actors can represent individuals, organizations, software, or hardware. According to the Big Data taxonomy, a single actor can play multiple roles, and multiple actors can play the same role. The NBDRA does not specify the business boundaries between the participating actors or stakeholders, so the roles can either reside within the same business entity or can be implemented by different business entities. Therefore, the NBDRA is applicable to a variety of business environments, from tightly integrated enterprise systems to loosely coupled vertical industries that rely on the cooperation of independent stakeholders. As a result, the notion of internal versus external functional components or roles does not apply to the NBDRA. However, for a specific use case, once the roles are associated with specific business stakeholders, the functional components would be considered as internal or externalsubject to the use case's point of view.

The NBDRA does support the representation of stacking or chaining of Big Data systems. For example, a Data Consumer of one system could serve as a Data Provider to the next system down the stack or chain.

The NBDRA is discussed in detail in the NBDIF: Volume 6, Reference Architecture. The Security and Privacy Fabric, and surrounding issues, are discussed in the NBDIF: Volume 4, Security and Privacy.

Once established, the definitions and reference architecture formed the basis for evaluation of existing standards to meet the unique needs of Big Data and evaluation of existing implementations and practices as candidates for new Big Data-related standards. In the first case, existing efforts may address standards gaps by either expanding or adding to the existing standard to accommodate Big Data characteristics or developing Big Data unique profiles within the framework of the existing standards. 


\section{BIG DATA STANDARDS}

Big Data has generated interest in a wide variety of multi-stakeholder, collaborative organizations. Some of the most involved to date have been organizations participating in the de jure standards process, industry consortia, and open source organizations. These organizations may operate differently and focus on different aspects, but they all have a stake in Big Data.

Integrating additional Big Data initiatives with ongoing collaborative efforts is a key to success. Identifying which collaborative initiative efforts address architectural requirements and which requirements are not currently being addressed is a starting point for building future multi-stakeholder collaborative efforts. Collaborative initiatives include, but are not limited to the following:

- Subcommittees and working groups of American National Standards Institute (ANSI);

- Accredited standards development organizations (SDOs; the de jure standards process);

- Industry consortia;

- Reference implementations; and

- Open source implementations.

Some of the leading SDOs and industry consortia working on Big Data-related standards include the following:

- IEC-International Electrotechnical Commission, http://www.iec.ch/;

- IEEE_-Institute of Electrical and Electronics Engineers, https://www.ieee.org/index.html, de jure standards process;

- IETF-Internet Engineering Task Force, https://www.ietf.org/;

- INCITS-International Committee for Information Technology Standards, http://www.incits.org/, de jure standards process;

- ISO-International Organization for Standardization, http://www.iso.org/iso/home.html, de jure standards process;

- OASIS-Organization for the Advancement of Structured Information Standards, https://www.oasis-open.org/, Industry consortium;

- $\quad$ OGC $^{\circledR}$ —Open Geospatial Consortium, http://www.opengeospatial.org/, Industry consortium;

- OGF-Open Grid Forum, https://www.ogf.org/ogf/doku.php, Industry consortium; and

- W3C-World Wide Web Consortium, http://www.w3.org/, Industry consortium.

The organizations and initiatives referenced in this document do not form an exhaustive list. It is anticipated that as this document is more widely distributed, more standards efforts addressing additional segments of the Big Data mosaic will be identified.

There are many government organizations that publish standards relative to their specific problem areas. The U.S. Department of Defense alone maintains hundreds of standards. Many of these are based on other standards (e.g., ISO, IEEE, ANSI) and could be applicable to the Big Data problem space. However, a fair, comprehensive review of these standards would exceed the available document preparation time and may not be of interest to most of the audience for this report. Readers interested in domains covered by the government organizations and standards, are encouraged to review the standards for applicability to their specific needs.

Open source implementations are providing useful new technologies used either directly or as the basis for commercially supported products. These open source implementations are not just individual products. Organizations will likely need to integrate an ecosystem of multiple products to accomplish 
their goals. Because of the ecosystem complexity and the difficulty of fairly and exhaustively reviewing open source implementations, many such implementations are not included in this section. However, it should be noted that those implementations often evolve to become the de facto reference implementations for many technologies.

\subsection{EXISTING STANDARDS}

The NBD-PWG embarked on an effort to compile a list of standards that are applicable to Big Data. The goal is to assemble Big Data-related standards that may apply to a large number of Big Data implementations across several domains. The enormity of the task precludes the inclusion of every standard that could apply to every Big Data implementation. Appendix B presents a partial list of existing standards from the above listed organizations that are relevant to Big Data and the NBDRA. Determining the relevance of standards to the Big Data domain is challenging since almost all standards in some way deal with data. Whether a standard is relevant to Big Data is generally determined by the impact of Big Data characteristics (i.e., volume, velocity, variety, and variability) on the standard or, more generally, by the scalability of the standard to accommodate those characteristics. A standard may also be applicable to Big Data depending on the extent to which that standard helps to address one or more of the Big Data characteristics. Finally, a number of standards are also very domain- or problem-specific and, while they deal with or address Big Data, they support a very specific functional domain; developing even a marginally comprehensive list of such standards would require a massive undertaking involving subject matter experts in each potential problem domain, which is currently beyond the scope of the NBD-PWG.

In selecting standards to include in Appendix B, the working group focused on standards that met the following criteria:

- Facilitate interfaces between NBDRA components;

- Facilitate the handling of data with one or more Big Data characteristics; and

- Represent a fundamental function needing to be implemented by one or more NBDRA components.

Appendix B represents a portion of potentially applicable standards from a portion of contributing organizations working in the Big Data domain.

As most standards represent some form of interface between components, the standards table in Appendix $C$ indicates whether the NBDRA component would be an Implementer or User of the standard. For the purposes of this table, the following definitions were used for Implementer and User.

Implementer: A component is an implementer of a standard if it provides services based on the standard (e.g., a service that accepts Structured Query Language [SQL] commands would be an implementer of that standard) or encodes or presents data based on that standard.

User: A component is a user of a standard if it interfaces to a service via the standard or if it accepts/consumes/decodes data represented by the standard.

While the above definitions provide a reasonable basis for some standards, the difference between implementation and use may be negligible or nonexistent.

\subsubsection{MAPPING EXISTING STANDARDS TO SPECIFIC REQUIREMENTS}

During Stage 2 work, the NBD-PWG began mapping the general requirements (Table 1) to applicable standards. Appendix A contains the entire Big Data standards catalog collected by the NBD-PWG to date. The requirements-to-standards matrix (Table 3) illustrates the mapping of the DCR category of general 
requirements to existing standards. The approach links a requirement with related standards by setting the requirement code and description in the same row as related standards descriptions and standards codes.

Table 3: Data Consumer Requirements-to-Standards Matrix

\begin{tabular}{ll|ll}
\hline Requirement & \multicolumn{1}{c|}{ Requirement Description } & \multicolumn{1}{c}{ Standard Description } & \multicolumn{1}{c}{ Standard } \\
\hline DCR-1 & Fast search & To be completed in version 3 & \\
DCR-2 & Diversified output file formats & To be completed in version 3 & \\
DCR-3 & $\begin{array}{l}\text { Visual layout of results for } \\
\text { presentation. }\end{array}$ & $\begin{array}{l}\text { Suggested charts and tables for } \\
\text { various purposes. }\end{array}$ & $\begin{array}{l}\text { International } \\
\text { Business } \\
\text { Communication }\end{array}$ \\
& & & $\begin{array}{l}\text { Standards } \\
\text { (IBCS) notation; } \\
\text { related: ACRL }\end{array}$ \\
DCR-4 & Browser access & & WebRTC \\
DCR-5 & Layer standard & To be completed in version 3 & ISO 13606 \\
\hline DCR-6 & Streaming results to clients & & \\
\hline
\end{tabular}

The work illustrated in Table 3 is representative of the work that should be continued with the other identified requirements groups (i.e., TPR, CPR, DCR, SPR, LMR, and OR) listed in Table 1 and explained fully in the NBDIF: Volume 3, Use Cases and General Requirements. The unpopulated requirements of DCR-1, DCR-2, and DCR-3 reflect only the unfinished nature of this topic, as of the date of this publication, due to limited available resources of the NBD-PWG, and should not be interpreted as standards gaps in the technology landscape. As more areas of the resulting matrix are completed, the matrix will provide a visual summary of the areas where standards overlap, and most importantly, highlight gaps in the standards catalog as of the date of publication.

\subsubsection{Mapping Existing Standards to SpeCIFIC Use Cases}

Similar to the standards to requirements mapping in Section 3.1.1, use cases were also mapped to standards (Table 4). Two use cases were initially selected for mapping and further analysis. These use cases were selected from the 51 version 1 use cases collected by the NBD-PWG and documented in the NBDIF: Volume 3, Use Cases and Requirements. The mapping illustrates the intersection of a domainspecific use case with standards related to Big Data. In addition, the mapping provides a visual summary of the areas where standards overlap and most importantly, highlights gaps in the standards catalog as of the date of publication of this document. The aim of the use case to standards mapping is to link a use case number and description with codes and descriptions for standards related to the use case. 
Table 4: General Mapping of Select Use Cases to Standards

\begin{tabular}{ll|ll}
\hline $\begin{array}{c}\text { Use Case Number } \\
\text { and Type }\end{array}$ & \multicolumn{1}{c|}{ Use Case Description } & Standard Description & \multicolumn{1}{c}{ Standard } \\
\hline 8: Commercial & Web search & $\begin{array}{l}\text { Xpath, Xquery } \\
\text { full-text, elixir, } \\
\text { xirql, xxl. }\end{array}$ \\
15: Defense & Intelligence data processing & $\begin{array}{l}\text { Collection of formats, } \\
\text { specifies Geo and Time } \\
\text { extensions, supports sharing } \\
\text { of search results }\end{array}$ & $\begin{array}{l}\text { OGC } \\
\text { OpenSearch }\end{array}$ \\
\hline
\end{tabular}

In addition to mapping standards that relate to the overall subject of a use case, specific portions of the original use cases (i.e., the categories of Current Solutions, Data Science, and Gaps) were mapped to standards. The detailed mapping provides additional granularity in the view of domain-specific standards. The data from the Current Solutions, Data Science, and Gaps categories, along with the subcategory data, was extracted from the raw use cases in the NBDIF: Volume 3, Use Cases and Requirements document. This data was tabulated with a column for standards related to each subcategory. The process of use case subcategory mapping was initiated with two use cases, Use Case 8 and Use Case 15, as evidenced below. The Standards Roadmap Subgroup might continue the process in version 3 of this document and requests the assistance of the public in this in-depth analysis.

\section{USE CASE 8: WEB SEARCH}

Table 5 demonstrates how the web search use case is divided into sub-task components and how related standards can be mapped to each sub-component.

Table 5: Excerpt from Use Case Document M0165—Detailed Mapping to Standards

\begin{tabular}{|c|c|c|c|}
\hline \multicolumn{3}{|c|}{ Information from Use Case 8} & \multirow{2}{*}{$\begin{array}{l}\text { Related } \\
\text { Standards }\end{array}$} \\
\hline Category & Subcategory & Use Case Data & \\
\hline \multirow{4}{*}{$\begin{array}{l}\text { Current } \\
\text { Solutions }\end{array}$} & Compute system & Large cloud & \\
\hline & Storage & Inverted index & \\
\hline & Networking & External most important & $\begin{array}{l}\text { SRU, SRW, CQL, Z39.50; } \\
\text { OAI PMH; Sparql, } \\
\text { representational state } \\
\text { transfer (REST), Href; }\end{array}$ \\
\hline & Software & & Spark (de facto) \\
\hline \multirow{5}{*}{$\begin{array}{l}\text { Data Science } \\
\text { (collection, } \\
\text { curation, } \\
\text { analysis, } \\
\text { action) }\end{array}$} & Veracity & Main hubs, authorities & \\
\hline & Visualization & $\begin{array}{l}\text { Page layout is critical. Technical elements inside a } \\
\text { website affect content delivery. }\end{array}$ & \\
\hline & Data Quality & & SRank \\
\hline & Data Types & & \\
\hline & Data Analytics & $\begin{array}{l}\text { Crawl, preprocess, index, rank, cluster, recommend. } \\
\text { Crawling / collection: connection elements including } \\
\text { mentions from other sites. }\end{array}$ & $\begin{array}{l}\text { Sitemap.xml, responsive } \\
\text { design (spec), }\end{array}$ \\
\hline Gaps & & Links to user profiles, social data & Schema.org \\
\hline
\end{tabular}




\section{USE CASE 15: Defense I NTELli genCe DATA PROCESSI ng AND ANALYSIS}

Table 6 demonstrates how the defense intelligence data processing use case is divided into sub-task components and how related standards can be mapped to each sub-component:

Table 6: Excerpt from Use Case Document M0215—Detailed Mapping to Standards

\begin{tabular}{|c|c|c|c|}
\hline \multicolumn{3}{|c|}{ Information from Use Case 15} & \multirow{2}{*}{$\begin{array}{l}\text { Related } \\
\text { Standards }\end{array}$} \\
\hline Category & Subcategory & Use Case Data & \\
\hline \multirow{4}{*}{$\begin{array}{l}\text { Current } \\
\text { Solutions }\end{array}$} & Compute system & $\begin{array}{l}\text { Fixed and deployed computing clusters ranging from } \\
1000 \text { s of nodes to } 10 \text { s of nodes. }\end{array}$ & \\
\hline & Storage & $\begin{array}{l}\text { Up to } 100 \text { s of PBs for edge and fixed site clusters. } \\
\text { Dismounted soldiers have at most 100s of GBs. }\end{array}$ & \\
\hline & Networking & $\begin{array}{l}\text { Connectivity to forward edge is limited and often high } \\
\text { latency and with packet loss. Remote communications } \\
\text { may be Satellite or limited to radio frequency / Line of } \\
\text { sight radio. }\end{array}$ & \\
\hline & Software & $\begin{array}{ll}\text { Currently baseline leverages: } \\
\text { 1. } \\
\text { 2. } & \text { Distributed storage } \\
\text { 3. } & \text { Natural Language Processing (NLP) } \\
\text { 4. } & \text { Deployment and security } \\
\text { 5. } & \text { Storm (spec) } \\
\text { 6. } & \text { Custom applications and visualization tools }\end{array}$ & $\begin{array}{l}\text { 1: Hadoop Distributed File } \\
\text { System (HDFS; de facto) } \\
\text { 3: GrAF (spec), } \\
\text { 4: Puppet (spec), }\end{array}$ \\
\hline \multirow{5}{*}{$\begin{array}{l}\text { Data Science } \\
\text { (collection, } \\
\text { curation, } \\
\text { analysis, } \\
\text { action) }\end{array}$} & $\begin{array}{l}\text { Veracity } \\
\text { (Robustness } \\
\text { Issues, } \\
\text { semantics) }\end{array}$ & $\begin{array}{l}\text { 1. Data provenance (e.g., tracking of all transfers and } \\
\text { transformations) must be tracked over the life of the } \\
\text { data. } \\
\text { 2. Determining the veracity of "soft" data sources } \\
\text { (generally human generated) is a critical requirement. }\end{array}$ & $\begin{array}{l}\text { 1: ISO/IEC 19763, W3C } \\
\text { Provenance }\end{array}$ \\
\hline & Visualization & $\begin{array}{l}\text { Primary visualizations will be Geospatial overlays and } \\
\text { network diagrams. Volume amounts might be millions of } \\
\text { points on the map and thousands of nodes in the } \\
\text { network diagram. }\end{array}$ & \\
\hline & $\begin{array}{l}\text { Data Quality } \\
\text { (syntax) }\end{array}$ & $\begin{array}{l}\text { Data Quality for sensor-generated data (image quality, } \\
\text { sig/noise) is generally known and good. } \\
\text { Unstructured or "captured" data quality varies } \\
\text { significantly and frequently cannot be controlled. }\end{array}$ & \\
\hline & Data Types & $\begin{array}{l}\text { Imagery, Video, Text, Digital documents of all types, } \\
\text { Audio, Digital signal data. }\end{array}$ & \\
\hline & Data Analytics & $\begin{array}{l}\text { 1. Near real time Alerts based on patterns and } \\
\text { baseline changes. } \\
\text { 2. Link Analysis } \\
\text { 3. Geospatial Analysis } \\
\text { 4. Text Analytics (sentiment, entity extraction, } \\
\text { etc.) }\end{array}$ & $\begin{array}{l}\text { 3: GeoSPARQL, } \\
\text { 4: SAML 2.0, }\end{array}$ \\
\hline Gaps & & $\begin{array}{l}\text { 1. Big (or even moderate size data) over tactical } \\
\text { networks } \\
\text { 2. Data currently exists in disparate silos which } \\
\text { must be accessible through a semantically } \\
\text { integrated data space. } \\
\text { 3. Most critical data is either unstructured or } \\
\text { imagery/video which requires significant } \\
\text { processing to extract entities and information. }\end{array}$ & $\begin{array}{l}\text { 1. } \\
\text { 2: SAML 2.0, } \\
\text { W3C OWL 2, } \\
\text { 3: }\end{array}$ \\
\hline
\end{tabular}




\subsection{MONITORING STANDARDS AS THEY EVOLVE}

Several pathways exist for the development of standards. The trajectory of this pathway is influenced by the SDO through which the standard is created and the domain to which the standard applies. For example, ANSI/ Standards Engineering Society (SES) 1:2012, Recommended Practice for the Designation and Organization of Standards, and SES 2:2011, Model Procedure for the Development of Standards, set forth documentation on how a standard itself must be defined.

Standards often evolve from requirements for certain capabilities. By definition, established de jure standards endorsed by official organizations, such as NIST, are ratified through structured procedures prior to the standard receiving a formal stamp of approval from the organization. The pathway from de jure standard to ratified standard often starts with a written deliverable that is given a Draft Recommendation status. If approved, the proposed standard then receives a higher Recommendation status, and continues up the ladder to a final status of Standard or perhaps International Standard.

Standards may also evolve from implementation of best practices and approaches which are proven against real-world applications, or from theory that is tuned to reflect additional variables and conditions uncovered during implementation. In contrast to formal standards that go through an approval process to meet the definition of ANSI/SES 1:2012, there are a range of technologies and procedures that have achieved a level of adoption in industry to become the conventional design in practice or method for practice, though they have not received formal endorsement from an official standards body. These dominant in-practice methods are often referred to as market-driven or de facto standards.

De facto standards may be developed and maintained in a variety of different ways. In proprietary environments, a single company will develop and maintain ownership of a de facto standard, in many cases allowing for others to make use of it. In some cases, this type of standard is later released from proprietary control into the Open Source environment. The open source environment also develops and maintains technologies of its own creation, while providing platforms for decentralized peer production and oversight on the quality of, and access to, the open source products.

The phase of development prior to the de facto standard is referred to as specifications. "When a tentative solution appears to have merit, a detailed written spec must be documented so that it can be implemented and codified.” [11]. Specifications must ultimately go through testing and pilot projects before reaching the next phases of adoption.

At the most immature end of the standards spectrum are the emerging technologies that are the result of $\mathrm{R} \& \mathrm{D}$. Here the technologies are the direct result of attempts to identify solutions to particular problems.

Since specifications and de facto standards can be very important to the development of Big Data systems, this volume attempts to include the most important standards and classify them appropriately.

Big Data efforts require a certain level of data quality. For example, metadata quality can be met using ISO 2709 (Implemented as MARC21) and thesaurus or ontology quality can be met by using ISO 25964.

In the case of Big Data, ANSI/NISO (National Information Standards Organization) has a number of relevant standards; many of these standards are also ISO Standards under ISO Technical Committee (TC) 46, which are Information and Documentation Standards. NISO and ISO TC 46 are working on addressing the requirements for Big Data standards through several committees and work groups.

U.S. federal departments and agencies are directed to use voluntary consensus standards developed by voluntary consensus standards bodies:

“'Voluntary consensus standards body' is a type of association, organization, or technical society that plans, develops, establishes, or coordinates voluntary consensus 
standards using a voluntary consensus standards development process that includes the following attributes or elements:

i. Openness: The procedures or processes used are open to interested parties. Such parties are provided meaningful opportunities to participate in standards development on a nondiscriminatory basis. The procedures or processes for participating in standards development and for developing the standard are transparent.

ii. Balance: The standards development process should be balanced. Specifically, there should be meaningful involvement from a broad range of parties, with no single interest dominating the decision making.

iii. Due process: Due process shall include documented and publicly available policies and procedures, adequate notice of meetings and standards development, sufficient time to review drafts and prepare views and objections, access to views and objections of other participants, and a fair and impartial process for resolving conflicting views.

iv. Appeals process: An appeals process shall be available for the impartial handling of procedural appeals.

v. Consensus: Consensus is defined as general agreement, but not necessarily unanimity. During the development of consensus, comments and objections are considered using fair, impartial, open, and transparent processes.” [12] 


\section{BIG DATA STANDARDS ROADMAP}

\subsection{GAPS IN STANDARDS}

A number of technology areas are considered to be of significant importance and are expected to have sizeable impacts heading into the next decade. Any list of important items will obviously not satisfy every community member; however, the potential gaps in Big Data standardization provided in this section describe broad areas that may be of interest to SDOs, consortia, and readers of this document.

The list below was produced through earlier work by an ISO/IEC Joint Technical Committee 1 (JTC1) Study Group on Big Data to serve as a potential guide to ISO in their establishment of Big Data standards activities. [13] The 16 potential Big Data standardization gaps, identified by the study group, described broad areas that may be of interest to this community. These gaps in standardization activities related to Big Data are in the following areas:

1. Big Data use cases, definitions, vocabulary, and reference architectures (e.g., system, data, platforms, online/offline);

2. Specifications and standardization of metadata including data provenance;

3. Application models (e.g., batch, streaming);

4. Query languages including non-relational queries to support diverse data types (e.g., XML, Resource Description Framework [RDF], JSON, multimedia) and Big Data operations (i.e., matrix operations);

5. Domain-specific languages;

6. Semantics of eventual consistency;

7. Advanced network protocols for efficient data transfer;

8. General and domain-specific ontologies and taxonomies for describing data semantics including interoperation between ontologies;

9. Big Data security and privacy access controls;

10. Remote, distributed, and federated analytics (taking the analytics to the data) including data and processing resource discovery and data mining;

11. Data sharing and exchange;

12. Data storage (e.g., memory storage system, distributed file system, data warehouse);

13. Human consumption of the results of Big Data analysis (e.g., visualization);

14. Energy measurement for Big Data;

15. Interface between relational (i.e., SQL) and non-relational (i.e., Not Only or No Structured Query Language [NoSQL]) data stores; and

16. Big Data quality and veracity description and management (includes master data management [MDM]).

Version 3 of this volume intends to investigate some of the 16 gaps identified above in further detail and may add more gaps in standardization activities to the list of 16 . The following sub-group of the 16 gaps was targeted for deeper analysis in Version 2 to explore individual issues of the gap and the impact future standards could have on the area.

- Gap 2: Specifications of metadata

- Gap 4: Non-relational database query, search and information retrieval (IR)

- Gap 10: Analytics

- Gap 11: Data sharing and exchange 


\subsection{PATHWAY TO ADDRESS GAPS IN STANDARDS}

The NBD-PWG Standards Roadmap Subgroup began an in-depth examination of the gaps presented in Section 4.1, including potential opportunities to close the gaps in standards. The first four gaps that were examined are presented in the following subsections.

\subsubsection{StandaRdS GaP 2: Metadata}

Metadata is one of the most significant of the Big Data problems. Metadata is the only way of finding items, yet $80 \%$ of data lakes are not applying metadata effectively. [14] Metadata layers are ways for lesser technical users to interact with data mining systems. Metadata layers also provide a means for bridging data stored in different locations, such as on premise and in the cloud. A definition and concept description of metadata is provided in the NBDIF: Volume 1, Definitions document.

Metadata issues have been addressed in ISO 2709-ANSI/NISO Z39.2 (implemented as MARC21) and cover not only metadata format but, using the related Anglo-American Cataloging Rules, content and input guidance for using the standard.

The metadata management field appears to now be converging with master data management [MDM] and somewhat also with analytics. Metadata management facilitates access control and governance, change management, and reduces complexity and the scope of change management, with the top use case likely to be data governance. [14] Demand for innovation in the areas of automating search capabilities such as semantic enrichment during load and inclusion of expert / community enrichment / crowd governance, and machine learning, remains strong and promises to continue.

Organizations that have existing metadata management systems will need to match any new metadata systems to the existing system, paying special attention to federation and integration issues. Organizations initiating new use cases or projects have much more latitude to investigate a range of potential solutions.

Perhaps a more attainable goal for standards development will be to strive for standards for supporting interoperability beyond the defining of ontologies, or XML, where investment of labor concentrates on the semantic mappings instead of syntactic mapping in smaller blocks that can be put together to form a larger picture, for example, to define conveying the semantics of who, what, where, and when of an event and translation of an individual user's terms (in order to create a module that can then be mapped to another standard).

\subsubsection{StandaRds Gap 4: Non-RELATIONAL DATABASE QUERY, SEARCH AND INFORMATION RETRIEVAL [IR]}

Search serves as a function for interfacing with data in both retrieval and analysis use cases. As a nonrelational database query function, search introduces a promise of self-service extraction capability over multiple sources of unstructured (and structured) Big Data in multiple internal and external locations. Search has capability to integrate with technologies for accepting natural language, and also for finding and analyzing patterns, statistics, and providing conceptual summary and consumable, visual formats.

This is an area where the ISO 23950/ANSI/NISO Z39.50 approach could help. From Wikipedia, "Z39.50 is an international standard client-server, application layer communications protocol for searching and retrieving information from a database over a Transmission Control Protocol / Internet Protocol (TCP/IP) computer network. It is covered by ANSI/NISO standard Z39.50, and ISO standard 23950.”

In that this is an age where one web search engine maintains the mindshare of the American public, it is important to clearly differentiate between the use of search as a data analysis method and the use of search for IR. Significantly different challenges are faced by business users undertaking search for 
information retrieval activities or using a search function for analysis of data that resides within an organization's storage repositories.

In web search, casual consumers are familiar with the experience of web search technologies, namely, instant query expansion, ranking of results, and rich snippets and knowledge graph containers. Casual users are also familiar with standard file folder functionality for information management in personal computers. For large enterprises and organizations needing search functionality over documents, deeper challenges persist and are driving significant demand for enterprise-grade solutions.

\section{Web Search}

Web search engines of 2017 provide a substantial service to citizens but have been identified as applying bias over how and what search results are delivered back to the user. The surrender of control that citizens willingly trade in exchange for the use of free web search services is widely accepted as a worthwhile exchange for the user; however, future technologies promise even more value for the citizens who will search across the rapidly expanding scale of the world wide web. The notable case in point is commonly referred to as the semantic web.

Current semantic approaches to searching almost all require content indexing as a measure for controlling the enormous corpus of documents that reside online. In attempting to tackle this problem of enormity of scale via automation of content indexing, solutions for the semantic web have proven to be difficult to program, meaning that the persistent challenges for development of a semantic web continue to delay its development.

Two promising approaches for developing the semantic web are ontologies and linked data technologies; however, neither approach has proven to be a complete solution. Standard Ontological alternatives, OWL and RDF, which would benefit from the addition of linked data, suffer from an inability to effectively use linked data technology. Reciprocally, linked data technologies suffer from the inability to effectively use ontologies.

Not apparent to developers is how standards in these areas would be an asset to the concept of an allencompassing semantic web, or how they can be integrated to improve retrieval over that scale of data.

\section{Using Search for Data Analysis}

A steady increase in the belief that logical search systems are the superior method for information retrieval on data at rest can be seen in the market. Generally speaking, analytics search indexes can be constructed more quickly than natural language processing (NLP) search systems, although NLP technologies requiring semi-supervision can have unacceptable (20\%) error rates.

Currently, Contextual Query Language (CQL) [15], declarative logic programming languages, and RDF [16] query languages currently serve as search query language / NoSQL language structure de facto standards.

Future work on this volume proposes to go deeper into discussing technologies' strengths in data acquisition, connectors, and ingest; and critical capabilities including speed and scale. For the most part, however, any product's underlying technology will likely be document, metadata, or numerically focused, not all three. Architecturally speaking, indexing is the centerpiece. Metadata provides context; machine learning can provide enrichment.

After indexing, query planning functionalities are of primary importance. The age of Big Data has applied a downward pressure on the use of standard indexes, which are good for small queries but have three issues: they cause slow loading; ad hoc queries require advance column indexing; and lastly, the constant updating that is required to maintain indexes quickly becomes prohibitively expensive. One open source search technology provides an incremental indexing technique that solves some part of this problem. 
Generally speaking, access and IR functions will remain areas of continual work in progress. In some cases, silo architectures for data are a necessary condition for running an organization, legal and security reasons being the most obvious. Proprietary, patented access methods are a barrier to building connectors required for true federated search. The future goal for many communities and enterprises in this area is the development of unified information access solutions (i.e., UIMA). Unified indexing presents an alternative to challenges in federation.

Incredibly valuable external data is underused in most search implementations because of the lack of an appropriate architecture. Frameworks that would separate content acquisition from content processing by putting a data buffer (a big copy of the data) between them have been suggested as a potential solution to this problem. With this framework, one could gather data but defer the content processing decisions until later. Documents would have to be pre-joined when they are processed for indexing, and large, mathematically challenging algorithms for relevancy and complex search security requirements (such as encryption) could be run separately at index time.

With such a framework, search could potentially become superior to SQL for online analytical processing (OLAP) and data warehousing. Search can be faster, more powerful, scalable, and schema free. Records can be output in XML and JSON and then loaded into a search engine. Fields can be mapped as needed.

Tensions remain between any given search system's functional power and its ease of use. Discovery, initially relegated to the limited functionality of facets in a sidebar, have historically been loaded when a search system returned a result set. Emerging technologies are focusing on supplementing user experience. Content Representation standards were initially relied upon in the Wide Area Information Servers (WAIS) system initially but newer systems must contend with the fact that there are now hundreds of formats. In response, open source technologies promise power and flexibility to customize, but the promise comes with a high price tag of either being technically demanding and requiring skilled staff to setup and operate, or requiring a third party to maintain.

Another area ripe for development is compatibility with different extract, transform, and load (ETL) techniques. Standards for connectors to content management systems, collaboration apps, web portals, social media apps, customer relationship management systems, file systems, and databases are needed.

Standards for content processing are still needed to enable compatibility with normalizing techniques, records merging formats, external taxonomies or semantic resources, regular expression, or use of metadata for supporting interface navigation functionality.

Standards for describing relationships between different data sources, and standards for maintaining metadata context relationships will have substantial impact. Semantic platforms to enhance information discovery and data integration applications may provide solutions in this area; RDF and ontology mapping seem to be the front runners in the race to provide semantic uniformity. RDF graphs are leading the way for visualization, and ontologies have become accepted methods for descriptions of elements.

\subsubsection{STANDARDS GAP 10: ANALYTICS}

Strictly speaking, analytics can be completed on small datasets without Big Data processing. The advent of more accessible tools, technologically and financially, for distributed computing and parallel processing of large datasets has had a profound impact on the discipline of analytics. Both the ubiquity of cloud computing and the availability of open source distributed computing tools have changed the way statisticians and data scientists perform analytics. Since the dawn of computing, scientists at national laboratories or large companies had access to the resources required to solve many computationally expensive and memory-intensive problems. Prior to Big Data, most statisticians did not have access to supercomputers and near-infinitely large databases. These technology limitations forced statisticians to consider trade-offs when conducting analyses and many times dictated which statistical learning model was applied. 
With the cloud computing revolution and the publication of open source tools to help setup and execute distributed computing environments, both the scope of analytics and the analytical methods available to statisticians changed, resulting in a new analytical landscape. This new analytical landscape left a gap in associated standards. Continual changes in the analytical landscape due to advances in Big Data technology are only worsening this standards gap.

Some examples of the changes to analytics due to Big Data are the following:

- Allowing larger and larger sample sizes to be processed and thus changing the power and sampling error of statistical results;

- Scaling out instead of scaling up, due to Big Data technology, has driven down the cost of storing large datasets;

- Increasing the speed of computationally expensive machine learning algorithms so that they are practical for analysis needs;

- Allowing in-memory analytics to achieve faster results;

- Allowing streaming or real-time analytics to apply statistical learning models in real time;

- Allowing enhanced visualization techniques for improved understanding;

- Cloud-based analytics made acquiring massive amounts of computing power for short periods of time financially accessible to businesses of all sizes and even individuals;

- Driving the creation of tools to make unstructured data appear structured for analysis;

- Shifting from an operational focus to an analytical focus with databases specifically designed for analytics;

- Allowing the analysis of more unstructured (NoSQL) data;

- Shifting the focus on scientific analysis from causation to correlation;

- Allowing the creation of data lakes, where the data model is not predefined prior to creation or analysis;

- Enhanced machine learning algorithms - training and test set sizes have been increased due to Big Data tools, leading to more accurate predictive models;

- Driving the analysis of behavioral data-Big Data tools have provided the computational capacity to analyze behavioral datasets such as web traffic or location data; and

- Enabling deep learning techniques.

With this new analytical landscape comes the need for additional knowledge beyond just statistical methods. Statisticians are required to have knowledge of which algorithms scale well and which algorithms deal with particular dataset sizes more efficiently.

For example, without Big Data tools, a random forest may be the best classification algorithm for a particular application provided project time constraints. However, with the computational resources afforded by Big Data, a deep learning algorithm may become the most accurate choice that satisfies the same project time constraints. Another prominent example is the selection of algorithms which handle streaming data well.

Standardizing analytical techniques and methodologies that apply to Big Data will have an impact on the accuracy, communicability, and overall effectiveness of analyses completed in accordance with this NBDIF.

\subsubsection{StandARDS GAP 11: DATA SHARING AND EXCHANGE}

The overarching goal of data sharing and exchange is to maximize access to data across heterogeneous repositories while still adhering to protect confidentiality and personal privacy. The objective is to improve the ability to locate and access digital assets such digital data, software, and publications while enabling proper long-term stewardship of these assets by optimizing archival functionality, and (where 
appropriate) leveraging existing institutional repositories, public and academic archives, as well as community and discipline-based repositories of scientific and technical data, software, and publications.

From the new global Internet, to Big Data economy opportunities in Internet of Things, smart cities, and other emerging technical and market trends, it is critical to have a standard data infrastructure for Big Data that is scalable and can apply the FAIR (Findability, Accessibility, Interoperability, and Reusability) data principle between heterogeneous datasets from various domains without worrying about data source and structure.

A very important component as part of the standard data infrastructure is the definition of new Persistent Identifier (PID) types. PIDs such as Digital Object Identifiers (DOIs) are already widely used on the Internet as durable, long-lasting references to digital objects such as publications or datasets. An obvious application of PIDs in this context is to use them to store a digital object's location and state information and other complex core metadata. In this way, the new PID types can serve to hold a combination of administration, specialized, and/or extension metadata. Other functional information, such as the properties and state of a repository or the types of access protocols it supports, can also be stored in these higher layers of PIDs.

Because the PIDs are themselves digital objects, they can be stored in specialized repositories, similar to metadata registries that can also expose services to digital object users and search portals. In this role, the PID types and the registries that manage them can be viewed as an abstraction layer in the system architecture, and could be implemented as middleware designed to optimize federated search, assist with access control, and speed the generation of cross-repository inventories. This setting can enable data integration/mashup among heterogeneous datasets from diversified domain repositories and make data discoverable, accessible, and usable through a machine-readable and actionable standard data infrastructure.

Organizations wishing to publish open data will find that there are certain legal constraints and licensing standards to be conscious of; data may not necessarily be 100\% Open in every sense of the word. There are, in fact, varying degrees to the openness of data; various licensing standards present a spectrum of licensing options, where each type allows for slightly differing levels of accommodations. Some licensing standards, including the Open Government License, provide truly open standards for data sharing.

Organizations wishing to publish open data must also be aware that there are some situations where the risks of having the data open, outweigh the benefits; and where certain licensing options are not appropriate, including situations when interoperability with other datasets is negatively affected. 


\section{INTEGRATION}

The term integration can refer to a broad range of activities or functions related to data processing. Those activities or functions can include systems integration or application integration middleware (business line communications processes), message queues, data integration, Application Programming Interfaces (APIs), or even continuous integration (i.e., code versioning). While the NBD-PWG respects the importance of all of these activities, not all activities are within the scope of this Version 2 of the NBDIF: Volume 7, Standards Roadmap. The following section focuses primarily on data integration and the most closely related architecture components. In this version of the Standards Roadmap data integration is viewed as the movement of data from source locations to target locations, and also the collection of information about what happened to the data during the data processing life cycle. Version three of the Standards Roadmap will explore deeper discussion on how integration is handled by multi-model database systems.

Big Data use cases brought about changes to the traditional data integration scenarios. Traditional data integration focused on the mechanics of moving structured data to or from different types of data structures via extraction from the source, transformation of that data into a format recognized by the target application, and then loading transformed data into the target application. Those ETL techniques historically configured separate tools for change data capture (CDC), replication, migration, and other ETL tasks. As the demand for additional capabilities required technologies with wider scopes, basic product lines in the ETL industry took on additional capabilities. Some technologies specialized in functions such as federation and data virtualization, synchronization, or data preparation. New companies that provided lightweight integration services through an integration platform as a service (iPaaS) model entered the market. By providing user-friendly features combined with cloud-technology advantages such as scalability, the agile iPaaS technologies enjoyed rapid adoption among organizations struggling with system integration requirements.

The most notable change to data integration approaches came in the form of a process where data was loaded immediately into a target location without any transformation; the transformation then took place inside the target system.

ETL is still important to data integration. However, with modern Big Data use cases, organizations are challenged to deal with unstructured data and data in motion, either of which results in a Big Data program requiring more attention to additional related systems such as MDM, synchronization, and data quality. [17] As such, there is a serious need for improved standardization in metadata and business rule management.

As of the publication date of this document, data integration is widely recognized as one of the primary elements required for leveraging Big Data environments. [14] 'Cutting edge' organizations are also shifting to architectures where the disparate integration implementations unify under a comprehensive umbrella for Big Data use cases.

Several integration topics are discussed in the remainder of this section. These are as follows:

- Data acquisition for data warehouses and analytics applications;

- Data cleansing;

- Data virtualization;

- Supporting master data management $[\mathrm{MDM}]$ and sharing metadata;

- Supporting governance (potential interoperability with mining, profiling, quality);

- Data migration; 
- Intra-organization and data consistency between apps, data warehouses, MDM;

- Inter-organizational data sharing;

- System integration, system consolidation, certified integration interfaces; and

- Metadata interfaces that provide nontechnical users with functionality for working with metadata (as a result of increasing importance of metadata).

\section{DATA ACQUISITION FOR DATA WAREHOUSES / ANALYTICS}

Connectivity is normally the first step in data processing, and support for all types of connections and all types of data are the dreams of Big Data users everywhere. Most off-the-shelf products offer a stable of connectors as part of the package. However, the 'usability' of a connector is just as important as the availability of the connector. The diversity of data types and data sources frequently means that custom middleware code must be written in order for a connector to work. Truly modern data acquisition designs provide easier-to-use graphic interfaces that abstract the complexities of programming a connector, away from the casual user. As the range of sources for data capture widens, the probability is greater that a more capable MDM or governance solution would be appropriate.

Aside from the types of data being captured, the modes of interaction or 'speed' of the data may dictate the type of integration required. The data warehouse is the traditional use case for data integration. In this scenario, large batches of transactions are extracted from a location point where they are at-rest, then processed in a single run that can take hours to complete. In some Big Data processing scenarios, users want immediate access to data that is streaming in-motion, so the system delivers results in real time, by capturing and processing small chunks of data within seconds. Real-time systems are more difficult to build and implement.

\section{DATA Cleansi ng}

Amidst most of the use cases for data integration is an absolute need to maximize data quality, which helps to ensure accuracy. Data must be cleaned to provide quality and accurate analytic outputs. This is especially true in cases where automated integration systems are in play.

One data cleansing design currently in practice promotes the creation of callable business rules, where, for example, the name and address attributes of a data record are checked upon data entry into an application, such as a customer relationship management system, which then uses custom exits to initiate a low-latency data quality process. This design requires hand-coded extensions for added flexibility over the base ETL tool, which must be carefully constructed to not violate the vendor's support of the base ETL tool.

Data preparation has been cited as consuming the majority of time and expense to process data. While quality is not mandatory for integration, it is commonly the most important element. Unstructured data is especially difficult to transform. Graphical interfaces, sometimes referred to as self-service interfaces, provide data preparation features which offer a promise of assisting business / casual users to explore data, transform and blend datasets, and perform analytics on top of a well-integrated infrastructure. The value of making data available to as many people as possible has been frequently noted.

\section{DATA VI RTUALIZATI ON}

Another area for consideration in Big Data systems implementation is that of data virtualization, or 'federation.' As one of the basic building blocks of a modern integration program, data virtualization is all about moving analysis to the data, in contrast to pulling data from a storage location into a data warehouse for analysis. Data virtualization programs are also applicable in small dataset data science scenarios.

\section{SUPPORTING MDM}


The boundaries between integration solutions and MDM solutions are increasingly blurred every year, with several functional sub-components having significant overlap. This makes sense if MDM is viewed as a quality function which is also a single point-of-truth concept for data entities.

Some current MDM tool designs use visual interfaces that allow everyone to use the same tool, see lineage and provenance of the processing, and reach a higher level of trust with the data. Using the same interface for system requirements gathering and translation to developers also reduces confusion in projects and increases the chance of successful implementations. Metadata management techniques are critical to MDM programs.

\section{SUPPORTI NG GOVERNANCE}

One perspective is that governance plays an integration role in the life cycle of Big Data, serving as the glue that binds the primary stages of the life cycle together. From this perspective, acquisition, awareness, and analytics of the data compose the full life cycle. The acquisition and awareness portions of this life cycle deal directly with data heterogeneity problems. Awareness, in this case, would generally be that the system, which acquires heterogeneous data from external sources, must have a contextual semantic framework (i.e., model) for integration of that data to make it usable.

The key areas where standards can promote the usability of data in this context are global resource identifiers, a model for storing data relationship classifications (such as RDF) and the creation of resource relationships. [18] Hence information architecture plays an increasingly important role. The awareness part of the cycle is also where the framework for identifying patterns in the data is constructed, and where metadata processing is managed. It is quite possible that this phase of the larger life cycle is the area most ready for innovation, although the analytics phase may be the part of the cycle currently undergoing the greatest transformation.

As the wrapper or glue that holds the parts of the Big Data life cycle together, a viable governance program will likely require a short list of properties for assuring the novelty, quality, utility, and validity of its data. As an otherwise equal partner in the Big Data life cycle, governance is not a technical function as the others, but rather more like a policy function that should reach into the cycle at all phases.

In some sense, governance issues present more serious challenges to organizations than other integration topics listed at the beginning of this section. Better data acquisition, consistency, sharing, and interfaces are highly desired. However, the mere mention of the term governance often induces thoughts of pain and frustration for an organization's management staff. Some techniques in the field have been found to have higher rates of end user acceptance and thus satisfaction of the organizational needs contained within the governance programs.

One of the more popular methods for improving governance-related standardization on datasets and reports is through a requirement that datasets and reports go through a review process that ensures that the data conforms to a handful of standards covering data ownership and aspects of IT. Upon passage of review, the data is given a 'watermark' which serves as an organization-wide seal of approval that the dataset or the report has been vetted and certified to be appropriate for sharing and decision making.

This process is popular partly because it is rather quick and easy to implement, minimizing push back from employees who must adopt this new process. The assessment for a watermark might include checks for appropriate or accurate calculations or metrics applied to the data, a properly structured dataset for additional processing, and application of proper permissions controls for supporting end user access. A data container, such as a data mart, can also serve as a form of data verification. [19]

\section{DATA MI GRATI ON}

The opportunity presented in data migration scenarios is to ensure data quality and, additionally, to clean and enrich the data to improve it during the migration process. A common-sense approach here is to apply business rules during the migration project, that leverage metadata to synchronize new data and update it 
as it is offloaded to a new system. Multi-model database technologies promise a reduction in the level of migration that is required for data processing.

\section{NTRA-ORGANI ZATI ON DATA CONSI STENCY, AND CROSS-SYSTEM DATA SYNCHRONIZATION}

With respect to consistency, this function can be thought of in terms of synchronization, which implies a synonymy with CDC. Batch CDC predates Big Data and is, therefore, not an area that deserves explication here. Although it may be interesting to note that metadata technologies can perform some CDC functionality.

Real-time CDC, however, is new to Big Data use cases and reflects a need for change broker or message queue technologies, which are ripe areas for standardization. Not surprisingly, data quality is an area of concern, as anyone can appreciate the unfortunate results if inaccurate data is propagated from one application within a department, across an entire enterprise. Best practices employ a CDC and message queue and trigger technology.

\section{NTER-ORGANI ZATI ONAL DATA SHARING}

The financial services, banking, and insurance (FSBI) sector has been an industry at the forefront of Big Data adoption. As such, FSBI can provide information about the challenges related to integration of external data sources. Due to the heterogeneous nature of external data, many resources are required for integrating external data with an organization's internal systems. In FSBI, the number of sources can also be high, creating a second dimension of difficulty.

By some reports [20], the lack of integration with internal systems is the largest organizational challenge when attempting to leverage external data sources. Many web portals and interfaces for external data sources do not provide APIs or capabilities that support automated integration, causing a situation where the majority of organizations currently relinquish expensive resources on manual coding methods to solve this problem. Of special interest in this area are designs offering conversion of SOAP protocol to REST (representational state transfer) protocol.

Aside from the expense, another problem with the hard coding methods is the resulting system inflexibility. Regardless of those challenges, the penalty for not integrating with external sources is even higher in the FSBI industry, where the issues of error and data quality are significant. The benefits of data validation and data integrity ultimately outweigh the costs.

As for describing APIs, one design promotes metadata descriptor calls that return an object's schema to the user, as well as all customer-created customizations, which are ideally based on controlled naming conventions for fields. This design also promotes service-level agreements (SLAs) providing contractual obligation that the provider will support specific API versions for lengths of time, as opposed to dropping support for previous API versions after release of new versions.

\section{SYSTEM I NTEGRATION}

One of the most important trends in systems integration involves what is referred to as hybrid integration. iPaaS solutions made particularly successful inroads into use cases for connecting on-premise systems to cloud applications (hybrid system integration), which is significant, because with Big Data more and more data lives in the cloud. The success of hybrid cloud technologies set the stage for the evolution of a newer category of technologies known as middleware as a service (MWaaS). MWaaS can be said to be based on API, business-to-business application integration, and cloud and fog system integration capabilities. As a consequence of the 'gravity' of data shifting to the cloud, MWaaS implementations are expected to make up larger shares of system integration programs in the near future. [21]

\section{MEtadATA}


Metadata is a pervasive requirement for integration programs and new standards for managing relationships between data sources; and automated discovery of metadata will be key to future Big Data projects.

In the worst cases, different departments within an organization often choose ETL tools without considering integration with other internal systems. This silo effect, coupled with the pooling of disparate systems that occurs after a business merger or acquisition, results in organizations that have several ETL tools in use that cannot interoperate. This situation often has a fragmenting effect on metadata programs as metadata cannot be exchanged. [17]

There are currently approximately 30 Metadata standards listed on the Digital Curation Centre (DCC) website (http://www.dcc.ac.uk/). Some of the lesser-known standards of a more horizontal data integration type are as follows:

- Data Package, version 1.0.0-beta.17 (a specification) released March of 2016;

- Observ-OM, integrated search. LGPLv3 Open Source licensed;

- PREMIS, independent serialization, preservation actor information;

- PROV, provenance information;

- QuDEx, agnostic formatting;

- Statistical Data and Metadata Exchange (SDMX), specification 2.1 last amended May of 2012; and

- $\quad$ Text Encoding and Interchange (TEI), varieties and modules for text encoding.

Recently, new technologies have emerged that analyze music, images, or video and generate metadata automatically. In the linked data community, efforts continue toward developing metadata techniques that automate construction of knowledge graphs and enable the inclusion of crowdsourced information. 


\section{Appendix A: Acronyms}

\begin{tabular}{|c|c|}
\hline ACRL & Association of College and Research Libraries \\
\hline AMQP & Advanced Message Queuing Protocol \\
\hline ANSI & American National Standards Institute \\
\hline API & Application Programming Interface \\
\hline AVC & Advanced Video Coding \\
\hline AVDL & Application Vulnerability Description Language \\
\hline BDAP & Big Data Application Provider \\
\hline BDFP & Big Data Framework Provider \\
\hline BIAS & Biometric Identity Assurance Services \\
\hline CCD & Continuity of Care Document \\
\hline CCR & Continuity of Care Record \\
\hline $\mathrm{CDC}$ & Change Data Capture \\
\hline CGM & Computer Graphics Metafile \\
\hline CIA & Confidentiality, Integrity, and Availability \\
\hline CMIS & Content Management Interoperability Services \\
\hline CPR & Capability Provider Requirements \\
\hline CQL & Contextual Query Language \\
\hline CTAS & Conformance Target Attribute Specification \\
\hline DC & Data Consumer \\
\hline DCAT & Data Catalog Vocabulary \\
\hline DCC & Digital Curation Centre \\
\hline DCR & Data Consumer Requirements \\
\hline DOI & Digital Object Identifier \\
\hline DOM & Document Object Model \\
\hline $\mathrm{DP}$ & Data Provider \\
\hline DSML & Directory Services Markup Language \\
\hline DSR & Data Source Requirements \\
\hline DSS & Digital Signature Service \\
\hline EPP & Extensible Provisioning Protocol \\
\hline ETL & Extract, Transform, Load \\
\hline EXI & Efficient XML Interchange \\
\hline FAIR & Findability, Accessibility, Interoperability, and Reusability \\
\hline FSBI & financial services, banking, and insurance \\
\hline GeoXACML & Geospatial eXtensible Access Control Markup Language \\
\hline GML & Geography Markup Language \\
\hline GRC & Governance, Risk management, and Compliance \\
\hline HDFS & Hadoop Distributed File System \\
\hline HEVC & High Efficiency Video Coding \\
\hline HITSP & Healthcare Information Technology Standards Panel \\
\hline HLVA & High-Level Version Architecture \\
\hline HTML & HyperText Markup Language \\
\hline HTTP & Hypertext Transfer Protocol \\
\hline IBCS & International Business Communication Standards \\
\hline IEC & International Electrotechnical Commission \\
\hline IEEE & Institute of Electrical and Electronics Engineers \\
\hline IETF & Internet Engineering Task Force \\
\hline
\end{tabular}




\begin{tabular}{|c|c|}
\hline INCITS & International Committee for Information Technology Standards \\
\hline iPaaS & integration platform as a service \\
\hline IR & Information Retrieval \\
\hline ISO & International Organization for Standardization \\
\hline IT & Information Technology \\
\hline ITL & Information Technology Laboratory \\
\hline ITS & Internationalization Tag Set \\
\hline JPEG & Joint Photographic Experts Group \\
\hline JSON & JavaScript Object Notation \\
\hline JSR & Java Specification Request \\
\hline JTC1 & Joint Technical Committee 1 \\
\hline LMR & Life Cycle Management Requirements \\
\hline $\mathrm{M}$ & Management Fabric \\
\hline MDM & Master Data Management \\
\hline MDX & Multidimensional expressions \\
\hline MFI & Metamodel Framework for Interoperability \\
\hline MOWS & Management of Web Services \\
\hline MPD & Model Package Description \\
\hline MPEG & Moving Picture Experts Group \\
\hline MQTT & Message Queuing Telemetry Transport \\
\hline MUWS & Management Using Web Services \\
\hline MWaaS & middleware as a service \\
\hline NARA & National Archives and Records Administration \\
\hline NASA & National Aeronautics and Space Administration \\
\hline NBD-PWG & NIST Big Data Public Working Group \\
\hline NBDIF & NIST Big Data Interoperability Framework \\
\hline NBDRA & NIST Big Data Reference Architecture \\
\hline NCAP & Network Capable Application Processor \\
\hline NCPDP & National Council for Prescription Drug Programs \\
\hline NDR & Naming and Design Rules \\
\hline netCDF & network Common Data Form \\
\hline NIEM & National Information Exchange Model \\
\hline NISO & National Information Standards Organization \\
\hline NIST & National Institute of Standards and Technology \\
\hline NLP & Natural Language Processing \\
\hline NoSQL & Not Only or No Structured Query Language \\
\hline NSF & National Science Foundation \\
\hline OASIS & Organization for the Advancement of Structured Information Standards \\
\hline OData & Open Data \\
\hline ODMS & On Demand Model Selection \\
\hline OGC & Open Geospatial Consortium \\
\hline OGF & Open Grid Forum \\
\hline OLAP & Online Analytical Processing \\
\hline OpenMI & Open Modelling Interface Standard \\
\hline OR & Other Requirements \\
\hline OWS Context & Web Services Context Document \\
\hline P3P & Platform for Privacy Preferences Project \\
\hline PICS & Platform for Internet Content Selection \\
\hline PID & Persistent Identifier \\
\hline PII & personally identifiable information \\
\hline PMML & Predictive modeling markup language \\
\hline
\end{tabular}


POWDER Protocol for Web Description Resources

RDF Resource Description Framework

REST representational state transfer

RFID Radio Frequency Identification

RIF Rule Interchange Format

RPM RedHat Package Manager

S\&P

SAF

SAML

SDMX

SDOs

SES

SFA

Security and Privacy Fabric

SKOS

SLAs

SML

SNMP

SO

SOAP

Symptoms Automation Framework

SPR

Security Assertion Markup Language

SQL

SWE

SWS

TC

TCP/IP

TEDS

Statistical Data and Metadata Exchange

Standards Development Organizations

Standards Engineering Society

Simple Features Access

Simple Knowledge Organization System Reference

TEI

TJS

TPR

TR

UBL

UDDI

UDP

UIMA

Service-Level Agreements

Service Modeling Language

Simple Network Management Protocol

System Orchestrator component

Simple Object Access Protocol

Security and Privacy Requirements

Structured Query Language

Sensor Web Enablement

Search Web Services

Technical Committee

UML

Transmission Control Protocol / Internet Protocol

Transducer Electronic Data Sheet

Text Encoding and Interchange

Table Joining Service

Transformation Provider Requirements

Technical Report

Universal Business Language

Universal Description, Discovery and Integration

User Datagram Protocol

Unstructured Information Management Architecture

UOML Unified Modeling Language

WAIS

Unstructured Operation Markup Language

W3C

Wide Area Information Servers

WCPS

World Wide Web Consortium

WCS Web Coverage Service

WebRTC Web Real-Time Communication

WFS

WMS

Web Feature Service

WPS

Web Map Service

WS-BPEL Web Services Business Process Execution Language

WS-Discovery Web Services Dynamic Discovery

WSDL Web Services Description Language

WSDM Web Services Distributed Management

WS-Federation Web Services Federation Language

WSN Web Services Notification

XACML eXtensible Access Control Markup Language 
NIST Big DATA INTEROPERABILITY FRAMEWORK: VOLUME 7, STANDARDS ROADMAP

XDM XPath Data Model

X-KISS XML Key Information Service Specification

XKMS XML Key Management Specification

X-KRSS XML Key Registration Service Specification

XMI

XML Metadata Interchange

XML

Extensible Markup Language

XSLT

Extensible Stylesheet Language Transformations 


\section{Appendix B: Collection of Big Data Related Standards}

The following table contains a collection of standards that pertain to a portion of the Big Data ecosystem. This collection is current, as of the date of publication of Volume 7. It is not an exhaustive list of standards that could relate to Big Data but rather a representative list of the standards that significantly impact some area of the Big Data ecosystem.

In selecting standards to include in Appendix B, the working group focused on standards that fit the following criteria:

- Facilitate interfaces between NBDRA components;

- Facilitate the handling of data with one or more Big Data characteristics; and

- Represent a fundamental function needing to be implemented by one or more NBDRA components.

Appendix B represents a portion of potentially applicable standards from a portion of contributing organizations working in Big Data domain.

\section{Table B-1: Big Data-Related Standards}

\begin{tabular}{ll}
\hline Standard Name/Number & Description \\
\hline ISO/IEC 9075-* & $\begin{array}{l}\text { ISO/IEC } 9075 \text { defines SQL. The scope of SQL is the definition of data structure and the operations on data stored } \\
\text { in that structure. ISO/IEC 9075-1, ISO/IEC 9075-2 and ISO/IEC 9075-11 encompass the minimum requirements } \\
\text { of the language. Other parts define extensions. }\end{array}$ \\
\hline ISO/IEC Technical Report (TR) 9789 Guidelines for the Organization and Representation of Data Elements for Data Interchange \\
$\begin{array}{l}\text { The } 11179 \text { standard is a multipart standard for the definition and implementation of Metadata Registries. The } \\
\text { series includes the following parts: } \\
\text { ISO/IEC 11179-* }\end{array}$ \\
$\begin{array}{l}\text { - Part 1: Framework } \\
\text { - Part 2: Classification }\end{array}$ \\
$\begin{array}{l}\text { - Part 4: Formulation of data definitions } \\
\text { - Part 5: Naming and identification principles }\end{array}$ \\
\hline ISO/IEC 10728-*
\end{tabular}




\section{Standard Name/Number}

ISO/IEC TR 19075-*

ISO/IEC 19503

ISO/IEC 19773

ISO/IEC TR 20943

ISO/IEC 19763-*

\section{Description}

This is a series of TRs on SQL related technologies.

- Part 1: Xquery

- Part 2: SQL Support for Time-Related Information

- Part 3: Programs Using the Java Programming Language

- Part 4: Routines and Types Using the Java Programming Language

Extensible Markup Language (XML) Metadata Interchange (XMI)

Metadata Registries Modules

Metadata Registry Content Consistency

Information Technology—Metamodel Framework for Interoperability (MFI) ISO/IEC 19763, Information

Technology -MFI. The 19763 standard is a multipart standard that includes the following parts:

- Part 1: Reference model

- Part 3: Metamodel for ontology registration

- Part 5: Metamodel for process model registration

- Part 6: Registry Summary

- Part 7: Metamodel for service registration

- Part 8: Metamodel for role and goal registration

- Part 9: On Demand Model Selection (ODMS) TR

- Part 10: Core model and basic mapping

- Part 12: Metamodel for information model registration

- Part 13: Metamodel for forms registration

- Part 14: Metamodel for dataset registration

- Part 15: Metamodel for data provenance registration

\begin{tabular}{|c|c|}
\hline ISO/IEC 9281:1990 & Information Technology—Picture Coding Methods \\
\hline ISO/IEC 10918:1994 & Information Technology—Digital Compression and Coding of Continuous-Tone Still Images \\
\hline ISO/IEC 11172:1993 & $\begin{array}{l}\text { Information Technology—Coding of Moving Pictures and Associated Audio for Digital Storage Media at up to } \\
\text { About 1,5 Mbit/s }\end{array}$ \\
\hline ISO/IEC 13818:2013 & Information Technology—Generic Coding of Moving Pictures and Associated Audio Information \\
\hline ISO/IEC 14496:2010 & Information Technology—Coding of Audio-Visual Objects \\
\hline ISO/IEC 15444:2011 & Information Technology_JPEG (Joint Photographic Experts Group) 2000 Image Coding System \\
\hline ISO/IEC 21000:2003 & Information Technology—Multimedia Framework (MPEG [Moving Picture Experts Group]-21) \\
\hline ISO 6709:2008 & Standard Representation of Geographic Point Location by Coordinates \\
\hline ISO 19115-* & Geographic Metadata \\
\hline ISO 19110 & Geographic Information Feature Cataloging \\
\hline
\end{tabular}




\begin{tabular}{|c|c|}
\hline Standard Name/Number & Description \\
\hline ISO 19139 & Geographic Metadata XML Schema Implementation \\
\hline ISO 19119 & Geographic Information Services \\
\hline ISO 19157 & Geographic Information Data Quality \\
\hline ISO 19114 & Geographic Information—Quality Evaluation Procedures \\
\hline IEEE 21451 -* & $\begin{array}{l}\text { Information Technology-Smart transducer interface for sensors and actuators } \\
\text { - Part 1: Network Capable Application Processor (NCAP) information model } \\
\text { - Part 2: Transducer to microprocessor communication protocols and Transducer Electronic Data Sheet } \\
\text { (TEDS) formats } \\
\text { - Part 4: Mixed-mode communication protocols and TEDS formats } \\
\text { Part 7: Transducer to radio frequency identification (RFID) systems communication protocols and TEDS } \\
\text { formats }\end{array}$ \\
\hline IEEE 2200-2012 & Standard Protocol for Stream Management in Media Client Devices \\
\hline ISO/IEC 15408-2009 & Information Technology—Security Techniques—Evaluation Criteria for IT Security \\
\hline ISO/IEC 27010:2012 & $\begin{array}{l}\text { Information Technology—Security Techniques-Information Security Management for Inter-Sector and Inter- } \\
\text { Organizational Communications }\end{array}$ \\
\hline ISO/IEC 27033-1:2009 & Information Technology—Security Techniques—Network Security \\
\hline ISO/IEC TR 14516:2002 & $\begin{array}{l}\text { Information Technology—Security Techniques—Guidelines for the Use and Management of Trusted Third-Party } \\
\text { Services }\end{array}$ \\
\hline ISO/IEC 29100:2011 & Information Technology—Security Techniques—Privacy Framework \\
\hline ISO/IEC 9798:2010 & Information Technology—Security Techniques—Entity Authentication \\
\hline ISO/IEC 11770:2010 & Information Technology—Security Techniques—Key Management \\
\hline ISO/IEC 27035:2011 & Information Technology_Security Techniques_-Information Security Incident Management \\
\hline ISO/IEC 27037:2012 & $\begin{array}{l}\text { Information Technology_Security Techniques—Guidelines for Identification, Collection, Acquisition and } \\
\text { Preservation of Digital Evidence }\end{array}$ \\
\hline $\begin{array}{l}\text { JSR (Java Specification Request) } 221 \\
\text { (developed by the Java Community } \\
\text { Process) }\end{array}$ & JDBCTM 4.0 Application Programming Interface (API) Specification \\
\hline W3C XML & XML 1.0 (Fifth Edition) W3C Recommendation 26 November 2008 \\
\hline $\begin{array}{l}\text { W3C Resource Description } \\
\text { Framework (RDF) }\end{array}$ & $\begin{array}{l}\text { The RDF is a framework for representing information in the Web. RDF graphs are sets of subject-predicate-object } \\
\text { triples, where the elements are used to express descriptions of resources. }\end{array}$ \\
\hline $\begin{array}{l}\text { W3C JavaScript Object Notation } \\
\text { (JSON)-LD } 1.0\end{array}$ & JSON-LD 1.0 A JSON-based Serialization for Linked Data W3C Recommendation 16 January 2014 \\
\hline
\end{tabular}




\section{Standard Name/Number}

W3C Document Object Model (DOM) Level 1 Specification

W3C XQuery 3.0

W3C XProc

W3C XML Encryption Syntax and Processing Version 1.1

W3C XML Signature Syntax and Processing Version 1.1

\section{W3C XPath 3.0}

W3C XSL Transformations (XSLT)

Version 2.0

W3C Efficient XML Interchange (EXI) Format 1.0 (Second Edition) W3C RDF Data Cube Vocabulary

W3C Data Catalog Vocabulary (DCAT)

W3C HTML5 A vocabulary and associated APIs for HTML and XHTML

W3C Internationalization Tag Set (ITS) 2.0

W3C OWL 2 Web Ontology Language

W3C Platform for Privacy Preferences (P3P) 1.0

\section{Description}

This series of specifications define the DOM, a platform- and language-neutral interface that allows programs and scripts to dynamically access and update the content, structure and style of HyperText Markup Language (HTML) and XML documents.

The XQuery specifications describe a query language called XQuery, which is designed to be broadly applicable across many types of XML data sources.

This specification describes the syntax and semantics of XProc: An XML Pipeline Language, a language for describing operations to be performed on XML documents.

This specification covers a process for encrypting data and representing the result in XML.

This specification covers XML digital signature processing rules and syntax. XML Signatures provide integrity, message authentication, and/or signer authentication services for data of any type, whether located within the XML that includes the signature or elsewhere.

XPath 3.0 is an expression language that allows the processing of values conforming to the data model defined in [XQuery and XPath Data Model (XDM) 3.0]. The data model provides a tree representation of XML documents as well as atomic values and sequences that may contain both references to nodes in an XML document and atomic values.

This specification defines the syntax and semantics of XSLT 2.0, a language for transforming XML documents into other XML documents.

This specification covers the EXI format. EXI is a very compact representation for the XML Information Set that is intended to simultaneously optimize performance and the utilization of computational resources.

The Data Cube vocabulary provides a means to publish multidimensional data, such as statistics on the Web using the W3C RDF standard.

DCAT is an RDF vocabulary designed to facilitate interoperability between data catalogs published on the Web. This document defines the schema and provides examples for its use.

This specification defines the 5th major revision of the core language of the World Wide Web-HTML.

The ITS 2.0 specification enhances the foundation to integrate automated processing of human language into core Web technologies and concepts that are designed to foster the automated creation and processing of multilingual Web content.

The OWL 2 Web Ontology Language, informally OWL 2, is an ontology language for the Semantic Web with formally defined meaning.

The P3P enables Web sites to express their privacy practices in a standard format that can be retrieved automatically and interpreted easily by user agents. 


\section{Standard Name/Number}

\section{Description}

W3C Protocol for Web Description Resources (POWDER)

W3C Provenance
POWDER — the Protocol for Web Description Resources—provides a mechanism to describe and discover Web resources and helps the users to decide whether a given resource is of interest.

Provenance is information about entities, activities, and people involved in producing a piece of data or thing, which can be used to form assessments about its quality, reliability or trustworthiness. The Provenance Family of Documents (PROV) defines a model, corresponding serializations and other supporting definitions to enable the inter-operable interchange of provenance information in heterogeneous environments such as the Web.

W3C Rule Interchange Format (RIF) RIF is a series of standards for exchanging rules among rule systems, in particular among Web rule engines.

W3C Service Modeling Language (SML) 1.1

W3C Simple Knowledge Organization System Reference (SKOS)

This specification defines the SML, Version 1.1 used to model complex services and systems, including their structure, constraints, policies, and best practices.

This document defines the SKOS, a common data model for sharing and linking knowledge organization systems via the Web.

W3C Simple Object Access Protocol (SOAP) 1.2

W3C SPARQL 1.1

SOAP is a protocol specification for exchanging structured information in the implementation of web services in computer networks.

SPARQL is a language specification for the query and manipulation of linked data in a RDF format.

W3C Web Service Description

Language (WSDL) 2.0

W3C XML Key Management

Specification (XKMS) 2.0

This standard specifies protocols for distributing and registering public keys, suitable for use in conjunction with the W3C Recommendations for XML Signature [XML-SIG] and XML Encryption [XML-Enc]. The XKMS comprises two parts:

- The XML Key Information Service Specification (X-KISS)

- The XML Key Registration Service Specification (X-KRSS).

OGC ${ }^{\circledR}$ OpenGIS ${ }^{\circledR}$ Catalogue Services Specification 2.0.2 -ISO Metadata This series of standard covers Catalogue Services based on ISO19115/ISO19119 are organized and implemented for the discovery, retrieval and management of data metadata, services metadata and application metadata.

The GeoAPI Standard defines, through the GeoAPI library, a Java language API including a set of types and methods which can be used for the manipulation of geographic information structured following the specifications adopted by the Technical Committee 211 of the ISO and by the OGC ${ }^{\circledR}$. 


\section{Standard Name/Number}

OGC ${ }^{\circledR}$ OpenGIS ${ }^{\circledR}$ GeoSPARQL

OGC $^{\circledR}$ OpenGIS ${ }^{\circledR}$ Geography Markup

Language (GML) Encoding Standard

OGC ${ }^{\circledR}$ Geospatial eXtensible Access Control Markup Language

(GeoXACML) Version 1

OGC $^{\circledR}$ network Common Data Form (netCDF)

OGC $^{\circledR}$ Open Modelling Interface

Standard (OpenMI)

OGC $^{\circledR}$ OpenSearch Geo and Time Extensions

OGC $^{\circledR}$ Web Services Context

Document (OWS Context)

OGC ${ }^{\circledR}$ Sensor Web Enablement (SWE)

OGC $^{\circledR}$ OpenGIS ${ }^{\circledR}$ Simple Features Access (SFA)

OGC $^{\circledR}$ OpenGIS ${ }^{\circledR}$ Georeferenced Table Joining Service (TJS) Implementation Standard

OGC $^{\circledR}$ OpenGIS ${ }^{\circledR}$ Web Coverage Processing Service Interface (WCPS)

Standard

OGC $^{\circledR}$ OpenGIS ${ }^{\circledR}$ Web Coverage Service (WCS)

\section{Description}

The OGC ${ }^{\circledR}$ GeoSPARQL standard supports representing and querying geospatial data on the Semantic Web. GeoSPARQL defines a vocabulary for representing geospatial data in RDF, and it defines an extension to the SPARQL query language for processing geospatial data.

The GML is an XML grammar for expressing geographical features. GML serves as a modeling language for geographic systems as well as an open interchange format for geographic transactions on the Internet.

The Policy Language introduced in this document defines a geo-specific extension to the XACML Policy Language, as defined by the OASIS standard eXtensible Access Control Markup Language (XACML), Version $2.0 ”$

netCDF is a set of software libraries and self-describing, machine-independent data formats that support the creation, access, and sharing of array-oriented scientific data.

The purpose of the OpenMI is to enable the runtime exchange of data between process simulation models and also between models and other modelling tools such as databases and analytical and visualization applications.

This OGC standard specifies the Geo and Time extensions to the OpenSearch query protocol. OpenSearch is a collection of simple formats for the sharing of search results.

The OGC ${ }^{\circledR}$ OWS Context was created to allow a set of configured information resources (service set) to be passed between applications primarily as a collection of services.

This series of standards support interoperability interfaces and metadata encodings that enable real time integration of heterogeneous sensor webs. These standards include a modeling language (SensorML), common data model, and sensor observation, planning, and alerting service interfaces.

Describes the common architecture for simple feature geometry and is also referenced as ISO 19125. It also implements a profile of the spatial schema described in ISO 19107:2003.

This standard is the specification for a TJS that defines a simple way to describe and exchange tabular data that contains information about geographic objects.

Defines a protocol-independent language for the extraction, processing, and analysis of multidimensional gridded coverages representing sensor, image, or statistics data.

This document specifies how a WCS offers multidimensional coverage data for access over the Internet. This document specifies a core set of requirements that a WCS implementation must fulfill. 


\section{Standard Name/Number Description}

OGC ${ }^{\circledR}$ Web Feature Service (WFS) 2.0 The WFS standard provides for fine-grained access to geographic information at the feature and feature property Interface Standard level. This International Standard specifies discovery operations, query operations, locking operations, transaction operations and operations to manage stored, parameterized query expressions.

OGC $^{\circledR}$ OpenGIS ${ }^{\circledR}$ Web Map Service

(WMS) Interface Standard

The OpenGIS ${ }^{\circ}$ WMS Interface Standard provides a simple HTTP (Hypertext Transfer Protocol) interface for requesting geo-registered map images from one or more distributed geospatial databases.

OGC ${ }^{\circledR}$ OpenGIS ${ }^{\circledR}$ Web Processing $\quad$ The OpenGIS ${ }^{\circledR}$ WPS Interface Standard provides rules for standardizing how inputs and outputs (requests and Service (WPS) Interface Standard responses) for geospatial processing services, such as polygon overlay. The standard also defines how a client can request the execution of a process, and how the output from the process is handled. It defines an interface that facilitates the publishing of geospatial processes and clients' discovery of and binding to those processes.

OASIS AS4 Profile of ebMS 3.0 v1.0

OASIS Advanced Message Queuing Protocol (AMQP) Version 1.0

OASIS Application Vulnerability

Description Language (AVDL) v1.0

OASIS Biometric Identity Assurance

Services (BIAS) Simple Object Access Protocol (SOAP) Profile v1.0

OASIS Content Management Interoperability Services (CMIS)

OASIS Digital Signature Service (DSS)

OASIS Directory Services Markup Language (DSML) v2.0

OASIS ebXML Messaging Services

OASIS ebXML RegRep
Standard for business to business exchange of messages via a web service platform.

The AMQP is an open internet protocol for business messaging. It defines a binary wire-level protocol that allows for the reliable exchange of business messages between two parties.

This specification describes a standard XML format that allows entities (such as applications, organizations, or institutes) to communicate information regarding web application vulnerabilities.

This OASIS BIAS profile specifies how to use XML (XML10) defined in ANSI INCITS 442-2010-BIAS to invoke SOAP -based services that implement BIAS operations.

The CMIS standard defines a domain model and set of bindings that include Web Services and ReSTful AtomPub that can be used by applications to work with one or more Content Management repositories/systems.

This specification describes two XML-based request/response protocols - a signing protocol and a verifying protocol. Through these protocols a client can send documents (or document hashes) to a server and receive back a signature on the documents; or send documents (or document hashes) and a signature to a server, and receive back an answer on whether the signature verifies the documents.

The DSML provides a means for representing directory structural information as an XML document methods for expressing directory queries and updates (and the results of these operations) as XML documents

These specifications define a communications-protocol neutral method for exchanging electronic business messages as XML.

ebXML RegRep is a standard defining the service interfaces, protocols and information model for an integrated registry and repository. The repository stores digital content while the registry stores metadata that describes the content in the repository. 


\section{Standard Name/Number}

OASIS ebXML Registry Information Model

\section{OASIS ebXML Registry Services}

Specification

\section{OASIS eXtensible Access Control} Markup Language (XACML)

OASIS Message Queuing Telemetry Transport (MQTT)

OASIS Open Data (OData) Protocol

OASIS Search Web Services (SWS)

OASIS Security Assertion Markup Language (SAML) v2.0

OASIS SOAP-over-UDP (User Datagram Protocol) v1.1

OASIS Solution Deployment Descriptor Specification v1.0

OASIS Symptoms Automation Framework (SAF) Version 1.0

OASIS Topology and Orchestration Specification for Cloud Applications Version 1.0

OASIS Universal Business Language (UBL) v2.1

\section{Description}

The Registry Information Model provides a blueprint or high-level schema for the ebXML Registry. It provides implementers with information on the type of metadata that is stored in the Registry as well as the relationships among metadata Classes.

An ebXML Registry is an information system that securely manages any content type and the standardized metadata that describes it. The ebXML Registry provides a set of services that enable sharing of content and metadata between organizational entities in a federated environment.

The standard defines a declarative access control policy language implemented in XML and a processing model describing how to evaluate access requests according to the rules defined in policies.

MQTT is a Client Server publish/subscribe messaging transport protocol for constrained environments such as for communication in Machine to Machine and Internet of Things contexts where a small code footprint is required and/or network bandwidth is at a premium.

The OData Protocol is an application-level protocol for interacting with data via RESTful interfaces. The protocol supports the description of data models and the editing and querying of data according to those models.

The OASIS SWS initiative defines a generic protocol for the interaction required between a client and server for performing searches. SWS define an Abstract Protocol Definition to describe this interaction.

The SAML defines the syntax and processing semantics of assertions made about a subject by a system entity. This specification defines both the structure of SAML assertions, and an associated set of protocols, in addition to the processing rules involved in managing a SAML system.

This specification defines a binding of SOAP to user datagrams, including message patterns, addressing requirements, and security considerations.

This specification defines schema for two XML document types: Package Descriptors and Deployment Descriptors. Package Descriptors define characteristics of a package used to deploy a solution. Deployment Descriptors define characteristics of the content of a solution package, including the requirements that are relevant for creation, configuration and maintenance of the solution content.

This standard defines reference architecture for the Symptoms Automation Framework, a tool in the automatic detection, optimization, and remediation of operational aspects of complex systems,

The concept of a "service template" is used to specify the "topology" (or structure) and "orchestration" (or invocation of management behavior) of IT services. This specification introduces the formal description of Service Templates, including their structure, properties, and behavior.

The OASIS UBL defines a generic XML interchange format for business documents that can be restricted or extended to meet the requirements of particular industries. 


\section{Standard Name/Number}

OASIS Universal Description, Discovery and Integration (UDDI) v3.0.2

OASIS Unstructured Information Management Architecture (UIMA) v1.0

OASIS Unstructured Operation Markup Language (UOML) v1.0 OASIS/W3C WebCGM v2.1

OASIS Web Services Business Process Execution Language (WS-BPEL) v2.0

OASIS/W3C - Web Services Distributed Management (WSDM): Management Using Web Services (MUWS) v1.1

OASIS WSDM: Management of Web Services (MOWS) v1.1

OASIS Web Services Dynamic Discovery (WS-Discovery) v1.1

OASIS Web Services Federation Language (WS-Federation) v1.2

\section{OASIS Web Services Notification} (WSN) v1.3

IETF Simple Network Management Protocol (SNMP) v3

\section{Description}

The focus of UDDI is the definition of a set of services supporting the description and discovery of (1) businesses, organizations, and other Web services providers, (2) the Web services they make available, and (3) the technical interfaces which may be used to access those services.

The UIMA specification defines platform-independent data representations and interfaces for text and multimodal analytics.

UOML is interface standard to process unstructured document; it plays the similar role as SQL to structured data. UOML is expressed with standard XML.

Computer Graphics Metafile (CGM) is an ISO standard, defined by ISO/IEC 8632:1999, for the interchange of $2 \mathrm{D}$ vector and mixed vector/raster graphics. WebCGM is a profile of CGM, which adds Web linking and is optimized for Web applications in technical illustration, electronic documentation, geophysical data visualization, and similar fields.

This standard defines a language for specifying business process behavior based on Web Services. WS-BPEL provides a language for the specification of Executable and Abstract business processes.

MUWS defines how an IT resource connected to a network provides manageability interfaces such that the IT resource can be managed locally and from remote locations using Web services technologies.

This part of the WSDM specification addresses management of the Web services endpoints using Web services protocols.

This specification defines a discovery protocol to locate services. The primary scenario for discovery is a client searching for one or more target services.

This specification defines mechanisms to allow different security realms to federate, such that authorized access to resources managed in one realm can be provided to security principals whose identities and attributes are managed in other realms.

WSN is a family of related specifications that define a standard Web services approach to notification using a topic-based publish/subscribe pattern.

SNMP is a series of IETF sponsored standards for remote management of system/network resources and transmission of status regarding network resources. The standards include definitions of standard management objects along with security controls. 


\section{Standard Name/Number $\quad$ Description}

IETF Extensible Provisioning Protocol This IETF series of standards describes an application-layer client-server protocol for the provisioning and (EPP) management of objects stored in a shared central repository. Specified in XML, the protocol defines generic object management operations and an extensible framework that maps protocol operations to objects.

National Council for Prescription Drug Programs (NCPDPD) Script standard ASTM Continuity of Care Record (CCR)

Healthcare Information Technology Standards Panel (HITSP) C32 HL7

Continuity of Care Document (CCD)

PMML Predictive Model Markup

Language

Dash7

H.265

VP9

Daala

WebRTC

X.509

MDX

NIEM-HLVA

NIEM-MPD

NIEM-Code List Specifications

NIEM Conformance Specification
Electronic data exchange standard used in medication reconciliation process. Medication history, prescription info (3), census update.

Electronic data exchange standard used in medication reconciliation process. CCR represents a summary format for the core facts of a patient's dataset.

Electronic data exchange standard used in medication reconciliation process. Summary format for CCR document structure.

XML based data handling. Mature standard defines and enables data modeling, and reliability and scalability for custom deployments. Pre / post processing, expression of predictive models.

Wireless sensor and actuator protocol; home automation, based on ISO IEC 18000-7

High efficiency video coding (HEVC) MPEG-H part 2. Potential compression successor to Advanced Video Coding (AVC) H.264. Streaming video.

Royalty free codec alternative to HEVC. Successor to VP8, competitor to H.265. Streaming video.

Video coding format. Streaming video.

Browser to browser communication

Public key encryption for securing email and web communication.

Multidimensional expressions (MDX) became the standard for OLAP query.

National Information Exchange Model (NIEM) High-Level Version Architecture (HLVA): Specifies the NIEM version architecture.

NIEM Model Package Description (MPD) Specification: Specifies rules for organizing and packaging MPDs in general and IEPDs specifically.

NIEM Code Lists Specification: Establishes methods for using code list artifacts with NIEM information exchange specifications.

Defines general conformance to NIEM. 


\section{Standard Name/Number}

NIEM-CTAS

\section{NIEM-NDR}

Non-Normative Guidance in Using NIEM with JSON

DCC Data Package, version 1.0.0beta.17 (a specification) released

March of 2016

DCC Observ-OM

Integrated search. LGPLv3 Open Source licensed

DCC PREMIS

DCC PROV

DCC QuDEx

DCC SDMX, specification 2.1 last

amended May of 2012

DCC TEI 2017.

Provenance information

Agnostic formatting

Varieties and modules for text encoding

\section{Description}

NIEM Conformance Target Attribute Specification (CTAS): Specifies XML attributes to establish a claim that the document conforms to a set of conformance targets.

NIEM Naming and Design Rules (NDR): Specifies principles and enforceable rules for NIEM-conformant schema documents, instance XML documents and data components.

Non-Normative Guidance in Using NIEM with JSON: Guidance for using NIEM with JSON-LD specified by RFC4627. Note: A normative NIEM-JSON specification is under development and scheduled for release in Dec

\section{Independent serialization, preservation actor information}




\section{Appendix C: Standards and the NBDRA}

As most standards represent some form of interface between components, the standards table in Appendix C indicates whether the NBDRA component would be an Implementer or User of the standard. For the purposes of this table, the following definitions were used for Implementer and User.

Implementer: A component is an implementer of a standard if it provides services based on the standard (e.g., a service that accepts Structured Query Language [SQL] commands would be an implementer of that standard) or encodes or presents data based on that standard.

User: A component is a user of a standard if it interfaces to a service via the standard or if it accepts/consumes/decodes data represented by the standard.

While the above definitions provide a reasonable basis for some standards, the difference between implementation and use may be negligible or nonexistent. The NBDRA components and fabrics are abbreviated in the table header as follows:

- $\mathrm{SO}=$ System Orchestrator

- $\mathrm{DP}=$ Data Provider

- $\quad \mathrm{DC}=$ Data Consumer

- $\quad \mathrm{BDAP}=$ Big Data Application Provider

- $\quad \mathrm{BDFP}=$ Big Data Framework Provider

- $\quad$ S\&P = Security and Privacy Fabric

- $\mathrm{M}=$ Management Fabric

Table C-1: Standards and the NBDRA

\begin{tabular}{|c|c|c|c|c|c|c|c|}
\hline \multirow[t]{2}{*}{ Standard Name/Number } & \multicolumn{7}{|c|}{ NBDRA Components } \\
\hline & SO & DP & DC & BDAP & BDFP & S\&P & M \\
\hline ISO/IEC 9075-* & & I & $\mathrm{I} / \mathrm{U}$ & $\mathrm{U}$ & $\mathrm{I} / \mathrm{U}$ & $\mathrm{U}$ & $\mathrm{U}$ \\
\hline ISO/IEC Technical Report (TR) 9789 & & $\mathrm{I} / \mathrm{U}$ & $\mathrm{I} / \mathrm{U}$ & $\mathrm{I} / \mathrm{U}$ & $\mathrm{I} / \mathrm{U}$ & & \\
\hline ISO/IEC 11179-* & & I & $\mathrm{I} / \mathrm{U}$ & $\mathrm{I} / \mathrm{U}$ & & $\mathrm{U}$ & \\
\hline ISO/IEC 10728-* & & & & & & & \\
\hline ISO/IEC 13249-* & & I & $\mathrm{I} / \mathrm{U}$ & U & $\mathrm{I} / \mathrm{U}$ & & \\
\hline ISO/IEC TR 19075-* & & I & $\mathrm{I} / \mathrm{U}$ & U & $\mathrm{I} / \mathrm{U}$ & & \\
\hline
\end{tabular}




\begin{tabular}{|c|c|c|c|c|c|c|c|}
\hline \multirow[t]{2}{*}{ Standard Name/Number } & \multicolumn{7}{|c|}{ NBDRA Components } \\
\hline & SO & DP & DC & BDAP & BDFP & S\&P & M \\
\hline ISO/IEC 19503 & & I & $\mathrm{I} / \mathrm{U}$ & $\mathrm{U}$ & $\mathrm{I} / \mathrm{U}$ & $\mathrm{U}$ & \\
\hline ISO/IEC 19773 & & I & $\mathrm{I} / \mathrm{U}$ & U & $\mathrm{I} / \mathrm{U}$ & $\mathrm{I} / \mathrm{U}$ & \\
\hline ISO/IEC TR 20943 & & I & $\mathrm{I} / \mathrm{U}$ & $\mathrm{U}$ & $\mathrm{I} / \mathrm{U}$ & $\mathrm{U}$ & $\mathrm{U}$ \\
\hline ISO/IEC 19763-* & & I & $\mathrm{I} / \mathrm{U}$ & $\mathrm{U}$ & $\mathrm{U}$ & & \\
\hline ISO/IEC 9281:1990 & & I & $\mathrm{U}$ & $\mathrm{I} / \mathrm{U}$ & $\mathrm{I} / \mathrm{U}$ & & \\
\hline ISO/IEC 10918:1994 & & I & $\mathrm{U}$ & $\mathrm{I} / \mathrm{U}$ & $\mathrm{I} / \mathrm{U}$ & & \\
\hline ISO/IEC 11172:1993 & & I & $\mathrm{U}$ & $\mathrm{I} / \mathrm{U}$ & $\mathrm{I} / \mathrm{U}$ & & \\
\hline ISO/IEC 13818:2013 & & I & $\mathrm{U}$ & $\mathrm{I} / \mathrm{U}$ & $\mathrm{I} / \mathrm{U}$ & & \\
\hline ISO/IEC 14496:2010 & & I & $\mathrm{U}$ & $\mathrm{I} / \mathrm{U}$ & $\mathrm{I} / \mathrm{U}$ & & \\
\hline ISO/IEC 15444:2011 & & I & $\mathrm{U}$ & $\mathrm{I} / \mathrm{U}$ & $\mathrm{I} / \mathrm{U}$ & & \\
\hline ISO/IEC 21000:2003 & & I & $\mathrm{U}$ & $\mathrm{I} / \mathrm{U}$ & $\mathrm{I} / \mathrm{U}$ & & \\
\hline ISO 6709:2008 & & I & $\mathrm{U}$ & $\mathrm{I} / \mathrm{U}$ & $\mathrm{I} / \mathrm{U}$ & & \\
\hline ISO 19115-* & & I & $\mathrm{U}$ & $\mathrm{I} / \mathrm{U}$ & $\mathrm{U}$ & & \\
\hline ISO 19110 & & I & $\mathrm{U}$ & $\mathrm{I} / \mathrm{U}$ & & & \\
\hline ISO 19139 & & I & $\mathrm{U}$ & $\mathrm{I} / \mathrm{U}$ & & & \\
\hline ISO 19119 & & I & $\mathrm{U}$ & $\mathrm{I} / \mathrm{U}$ & & & \\
\hline ISO 19157 & & I & $\mathrm{U}$ & $\mathrm{I} / \mathrm{U}$ & $\mathrm{U}$ & & \\
\hline ISO 19114 & & & & I & & & \\
\hline IEEE 21451 -* & & I & $\mathrm{U}$ & & & & \\
\hline IEEE 2200-2012 & & I & $\mathrm{U}$ & $\mathrm{I} / \mathrm{U}$ & & & \\
\hline ISO/IEC 15408-2009 & $\mathrm{U}$ & & & & & I & \\
\hline ISO/IEC 27010:2012 & & I & $\mathrm{U}$ & $\mathrm{I} / \mathrm{U}$ & & & \\
\hline ISO/IEC 27033-1:2009 & & $\mathrm{I} / \mathrm{U}$ & $\mathrm{I} / \mathrm{U}$ & $\mathrm{I} / \mathrm{U}$ & I & & \\
\hline ISO/IEC TR 14516:2002 & $\mathrm{U}$ & & & & & $\mathrm{U}$ & \\
\hline ISO/IEC 29100:2011 & & & & & & I & \\
\hline ISO/IEC 9798:2010 & & $\mathrm{I} / \mathrm{U}$ & $\mathrm{U}$ & $\mathrm{U}$ & $\mathrm{U}$ & $\mathrm{I} / \mathrm{U}$ & \\
\hline ISO/IEC 11770:2010 & & $\mathrm{I} / \mathrm{U}$ & $\mathrm{U}$ & $\mathrm{U}$ & $\mathrm{U}$ & $\mathrm{I} / \mathrm{U}$ & \\
\hline ISO/IEC 27035:2011 & $\mathrm{U}$ & & & & & I & \\
\hline ISO/IEC 27037:2012 & $\mathrm{U}$ & & & & & I & \\
\hline JSR (Java Specification Request) 221 (developed by the Java Community Process) & & $\mathrm{I} / \mathrm{U}$ & $\mathrm{I} / \mathrm{U}$ & $\mathrm{I} / \mathrm{U}$ & $\mathrm{I} / \mathrm{U}$ & & \\
\hline
\end{tabular}




\begin{tabular}{|c|c|c|c|c|c|c|c|}
\hline \multirow[t]{2}{*}{ Standard Name/Number } & \multicolumn{7}{|c|}{ NBDRA Components } \\
\hline & SO & DP & DC & BDAP & BDFP & S\&P & M \\
\hline W3C XML & $\mathrm{I} / \mathrm{U}$ & $\mathrm{I} / \mathrm{U}$ & $\mathrm{I} / \mathrm{U}$ & $\mathrm{I} / \mathrm{U}$ & $\mathrm{I} / \mathrm{U}$ & $\mathrm{I} / \mathrm{U}$ & $\mathrm{I} / \mathrm{U}$ \\
\hline W3C Resource Description Framework (RDF) & & I & $\mathrm{U}$ & $\mathrm{I} / \mathrm{U}$ & $\mathrm{I} / \mathrm{U}$ & & \\
\hline W3C JavaScript Object Notation (JSON)-LD 1.0 & & I & U & $\mathrm{I} / \mathrm{U}$ & $\mathrm{I} / \mathrm{U}$ & & \\
\hline W3C Document Object Model (DOM) Level 1 Specification & & I & U & $\mathrm{I} / \mathrm{U}$ & $\mathrm{I} / \mathrm{U}$ & & \\
\hline W3C XQuery 3.0 & & I & U & $\mathrm{I} / \mathrm{U}$ & $\mathrm{I} / \mathrm{U}$ & & \\
\hline W3C XProc & I & I & U & $\mathrm{I} / \mathrm{U}$ & $\mathrm{I} / \mathrm{U}$ & & \\
\hline W3C XML Encryption Syntax and Processing Version 1.1 & & I & $\mathrm{U}$ & $\mathrm{I} / \mathrm{U}$ & & & \\
\hline W3C XML Signature Syntax and Processing Version 1.1 & & I & U & $\mathrm{I} / \mathrm{U}$ & & & \\
\hline W3C XPath 3.0 & & I & U & $\mathrm{I} / \mathrm{U}$ & $\mathrm{I} / \mathrm{U}$ & & \\
\hline W3C XSL Transformations (XSLT) Version 2.0 & & I & $\mathrm{U}$ & $\mathrm{I} / \mathrm{U}$ & $\mathrm{I} / \mathrm{U}$ & & \\
\hline W3C Efficient XML Interchange (EXI) Format 1.0 (Second Edition) & & I & $\mathrm{U}$ & $\mathrm{I} / \mathrm{U}$ & & & \\
\hline W3C RDF Data Cube Vocabulary & & I & $\mathrm{U}$ & $\mathrm{I} / \mathrm{U}$ & $\mathrm{I} / \mathrm{U}$ & & \\
\hline W3C Data Catalog Vocabulary (DCAT) & & I & $\mathrm{U}$ & $\mathrm{I} / \mathrm{U}$ & & & \\
\hline W3C HTML5 A vocabulary and associated APIs for HTML and XHTML & & I & $\mathrm{U}$ & $\mathrm{I} / \mathrm{U}$ & & & \\
\hline W3C Internationalization Tag Set (ITS) 2.0 & & I & $\mathrm{U}$ & $\mathrm{I} / \mathrm{U}$ & $\mathrm{I} / \mathrm{U}$ & & \\
\hline W3C OWL 2 Web Ontology Language & & I & $\mathrm{U}$ & $\mathrm{I} / \mathrm{U}$ & $\mathrm{I} / \mathrm{U}$ & & \\
\hline W3C Platform for Privacy Preferences (P3P) 1.0 & & I & $\mathrm{U}$ & $\mathrm{I} / \mathrm{U}$ & & $\mathrm{I} / \mathrm{U}$ & \\
\hline W3C Protocol for Web Description Resources (POWDER) & & I & $\mathrm{U}$ & $\mathrm{I} / \mathrm{U}$ & & & \\
\hline W3C Provenance & & I & $\mathrm{U}$ & $\mathrm{I} / \mathrm{U}$ & $\mathrm{I} / \mathrm{U}$ & $\mathrm{U}$ & \\
\hline W3C Rule Interchange Format (RIF) & & I & U & $\mathrm{I} / \mathrm{U}$ & $\mathrm{I} / \mathrm{U}$ & & \\
\hline W3C Service Modeling Language (SML) 1.1 & $\mathrm{I} / \mathrm{U}$ & I & $\mathrm{U}$ & $\mathrm{I} / \mathrm{U}$ & & & \\
\hline W3C Simple Knowledge Organization System Reference (SKOS) & & I & $\mathrm{U}$ & $\mathrm{I} / \mathrm{U}$ & & & \\
\hline W3C Simple Object Access Protocol (SOAP) 1.2 & & I & $\mathrm{U}$ & $\mathrm{I} / \mathrm{U}$ & & & \\
\hline W3C SPARQL 1.1 & & I & $\mathrm{U}$ & $\mathrm{I} / \mathrm{U}$ & $\mathrm{I} / \mathrm{U}$ & & \\
\hline W3C Web Service Description Language (WSDL) 2.0 & $\mathrm{U}$ & I & $\mathrm{U}$ & $\mathrm{I} / \mathrm{U}$ & & & \\
\hline W3C XML Key Management Specification (XKMS) 2.0 & $\mathrm{U}$ & I & $\mathrm{U}$ & $\mathrm{I} / \mathrm{U}$ & & & \\
\hline OGC $^{\circledR}$ OpenGIS ${ }^{\circledR}$ Catalogue Services Specification 2.0.2 - & & I & $\mathrm{U}$ & $\mathrm{I} / \mathrm{U}$ & & & \\
\hline \multicolumn{8}{|l|}{ ISO Metadata Application Profile } \\
\hline OGC $^{\circledR}$ OpenGIS ${ }^{\circledR}$ GeoAPI & & I & $\mathrm{U}$ & $\mathrm{I} / \mathrm{U}$ & $\mathrm{I} / \mathrm{U}$ & & \\
\hline OGC $^{\circledR}$ OpenGIS ${ }^{\circledR}$ GeoSPARQL & & I & $\mathrm{U}$ & $\mathrm{I} / \mathrm{U}$ & $\mathrm{I} / \mathrm{U}$ & & \\
\hline
\end{tabular}


OGC ${ }^{\circledR}$ OpenGIS ${ }^{\circledR}$ Geography Markup Language (GML) Encoding Standard

OGC $^{\circledR}$ Geospatial eXtensible Access Control Markup Language (GeoXACML)

Version 1

OGC ${ }^{\circledR}$ network Common Data Form (netCDF)

OGC ${ }^{\circledR}$ Open Modelling Interface Standard (OpenMI)

OGC ${ }^{\circledR}$ OpenSearch Geo and Time Extensions

OGC ${ }^{\circledR}$ Web Services Context Document (OWS Context)

OGC ${ }^{\circledR}$ Sensor Web Enablement (SWE)

OGC $^{\circledR}$ OpenGIS ${ }^{\circledR}$ Simple Features Access (SFA)

OGC $^{\circledR}$ OpenGIS ${ }^{\circledR}$ Georeferenced Table Joining Service (TJS) Implementation

Standard

OGC ${ }^{\circledR}$ OpenGIS ${ }^{\circledR}$ Web Coverage Processing Service Interface (WCPS) Standard

OGC $^{\circledR}$ OpenGIS ${ }^{\circledR}$ Web Coverage Service (WCS)

OGC ${ }^{\circledR}$ Web Feature Service (WFS) 2.0 Interface Standard

OGC ${ }^{\circledR}$ OpenGIS ${ }^{\circledR}$ Web Map Service (WMS) Interface Standard

OGC $^{\circledR}$ OpenGIS ${ }^{\circledR}$ Web Processing Service (WPS) Interface Standard

OASIS AS4 Profile of ebMS 3.0 v1.0

OASIS Advanced Message Queuing Protocol (AMQP) Version 1.0

OASIS Application Vulnerability Description Language (AVDL) v1.0

OASIS Biometric Identity Assurance Services (BIAS) Simple Object Access

Protocol (SOAP) Profile v1.0

OASIS Content Management Interoperability Services (CMIS)

OASIS Digital Signature Service (DSS)

OASIS Directory Services Markup Language (DSML) v2.0

OASIS ebXML Messaging Services

OASIS ebXML RegRep

OASIS ebXML Registry Information Model

OASIS ebXML Registry Services Specification

OASIS eXtensible Access Control Markup Language (XACML)

OASIS Message Queuing Telemetry Transport (MQTT)

OASIS Open Data (OData) Protocol 
OASIS Search Web Services (SWS)

OASIS Security Assertion Markup Language (SAML) v2.0

OASIS SOAP-over-UDP (User Datagram Protocol) v1.1

OASIS Solution Deployment Descriptor Specification v1.0

OASIS Symptoms Automation Framework (SAF) Version 1.0

OASIS Topology and Orchestration Specification for Cloud Applications Version 1.0

OASIS Universal Business Language (UBL) v2.1

OASIS Universal Description, Discovery and Integration (UDDI) v3.0.2

OASIS Unstructured Information Management Architecture (UIMA) v1.0

OASIS Unstructured Operation Markup Language (UOML) v1.0

OASIS/W3C WebCGM v2.1

OASIS Web Services Business Process Execution Language (WS-BPEL) v2.0

OASIS/W3C - Web Services Distributed Management (WSDM): Management Using Web Services (MUWS) v1.1

OASIS WSDM: Management of Web Services (MOWS) v1.1

OASIS Web Services Dynamic Discovery (WS-Discovery) v1.1

OASIS Web Services Federation Language (WS-Federation) v1.2

OASIS Web Services Notification (WSN) v1.3

IETF Simple Network Management Protocol (SNMP) v3

IETF Extensible Provisioning Protocol (EPP)

NCPDPD Script standard

ASTM Continuity of Care Record (CCR) message

Healthcare Information Technology Standards Panel (HITSP) C32 HL7 Continuity of

Care Document (CCD)

PMML Predictive Model Markup Language

Dash7

H.265

VP9

Daala

WebRTC

X.509 
NIST BIG DATA INTEROPERABILITY FRAMEWORK: VOLUME 7, STANDARDS ROADMAP

\begin{tabular}{|c|c|c|c|c|c|c|c|}
\hline Standard Name/Number & \multicolumn{7}{|c|}{ NBDRA Components } \\
\hline MDX & $\mathrm{SO}$ & DP & DC & BDAP & BDFP & S\&P & M \\
\hline NIEM-HLVA & & $\mathrm{I} / \mathrm{U}$ & $\mathrm{I} / \mathrm{U}$ & $\mathrm{I} / \mathrm{U}$ & & & \\
\hline NIEM-MPD & & $\mathrm{I} / \mathrm{U}$ & $\mathrm{I} / \mathrm{U}$ & $\mathrm{I} / \mathrm{U}$ & & & \\
\hline NIEM-Code List Specifications & & $\mathrm{I} / \mathrm{U}$ & $\mathrm{I} / \mathrm{U}$ & $\mathrm{I} / \mathrm{U}$ & & & \\
\hline NIEM Conformance Specification & & $\mathrm{I} / \mathrm{U}$ & $\mathrm{I} / \mathrm{U}$ & $\mathrm{I} / \mathrm{U}$ & & & \\
\hline NIEM-CTAS & & $\mathrm{I} / \mathrm{U}$ & $\mathrm{I} / \mathrm{U}$ & $\mathrm{I} / \mathrm{U}$ & & & \\
\hline NIEM-NDR & & $\mathrm{I} / \mathrm{U}$ & $\mathrm{I} / \mathrm{U}$ & $\mathrm{I} / \mathrm{U}$ & & & \\
\hline Non-Normative Guidance in Using NIEM with JSON & & $\mathrm{I} / \mathrm{U}$ & $\mathrm{I} / \mathrm{U}$ & $\mathrm{I} / \mathrm{U}$ & & & \\
\hline DCC Data Package, version 1.0.0-beta.17 (a specification) & & & & & & & \\
\hline DCC Observ-OM $\backslash$ & & & & & & & \\
\hline DCC PREMIS & & & & & & & \\
\hline DCC PROV & & & & & & & \\
\hline DCC QuDEx & & & & & & & \\
\hline DCC SDMX, specification 2.1 last amended May of 2012 & & & & & & & \\
\hline DCC TEI & & & & & & & \\
\hline
\end{tabular}




\section{Appendix D: Categorized Standards}

Large catalogs of standards, such as the collection in Appendix B and C, describe the characteristics and relevance of existing standards. In the catalog format presented in Appendix D, the NBD-PWG strives to provide a structure for an ongoing process that supports continuous improvement of the catalog to ensure the usefulness of it in the years to come, even as technologies and requirements evolve over time.

The approach is to identify standards with one or more category terms, allowing readers to cross-reference the list of standards either by application domains or classes of activities defined in the NBDRA. The categorized standards could help to reduce the long list of standards to a shorter list that is relevant to the reader's area of concern.

Additional contributions from the public are invited. Please see the Request for Contribution in the front matter of this document for methods to submit contributions. First, contributors can identify standards that relate to application domains and NBDRA activities category terms and fill in the columns in Table E-1. Second, additional categorization columns could be suggested, which should contain classification terms and should be broad enough to apply to a majority of readers.

The application domains and NBDRA activities defined to date are listed below. Additional information on the selection of application domains is contained in the NBDIF: Volume 3, Use Cases and Requirements. The NBDIF: Volume 6, Reference Architecture expounds on the NBDRA activities.

\section{Application domains defined to date:}

- Government Operations

- Commercial

- Defense

- Healthcare and Life Sciences

- Deep Learning and Social Media

- The Ecosystem for Research

- Astronomy and Physics

- Earth, Environmental and Polar Science

- Energy

- IoT

- Multimedia 


\section{NBDRA classes of activities defined to date:}

\section{- System Orchestrator (SO)}

o Business Ownership Requirements and Monitoring

o Governance Requirements and Monitoring

o System Architecture Requirements Definition

o Data Science Requirements and Monitoring

o Security/Privacy Requirements Definition and Monitoring

\section{- Big Data Framework Provider (BDFP)}

o Messaging

o Resource Management

o Processing: Batch Processing

o Processing: Interactive Processing

o Processing: Stream Processing

o Platforms: Create

o Platforms: Read

o Platforms: Update

o Platforms: Delete

o Platforms: Index

o Infrastructures: Transmit

o Infrastructures: Receive

o Infrastructures: Store

o Infrastructures: Manipulate

o Infrastructures: Retrieve

\section{- Security and Privacy (SP)}

o Authentication

o Authorization

o Auditing

- Management (M)
o Provisioning
o Configuration
o Package Management
o Resource Management
o Monitoring

- Big Data Application Provider (BDAP)
o Collection
o Preparation
o Analytics
o Visualization
o Access

Whereas the task of categorization is immense and resources are limited, completion of this table relies on new and renewed contributions from the public. The NBD-PWG invites all interested parties to assist in the categorization effort.

\section{Table D-1: Categorized Standards}


Standard Name/Number

ISO/IEC 10728-*

ISO/IEC 13249-*

ISO/IEC TR 19075-*

ISO/IEC 19503

ISO/IEC 19773

ISO/IEC TR 20943

ISO/IEC 19763-*

ISO/IEC 9281:1990

ISO/IEC 10918:1994

ISO/IEC 11172:1993

ISO/IEC 13818:2013

ISO/IEC 14496:2010

ISO/IEC 15444:2011

ISO/IEC 21000:2003

ISO 6709:2008

ISO 19115-*

ISO 19110

ISO 19139

ISO 19119

ISO 19157

ISO 19114

IEEE 21451 -*

IEEE 2200-2012

ISO/IEC 15408-2009

ISO/IEC 27010:2012

ISO/IEC 27033-1:2009

ISO/IEC TR 14516:2002

ISO/IEC 29100:2011

ISO/IEC 9798:2010

ISO/IEC 11770:2010

ISO/IEC 27035:2011

\section{Application Domain}

NBDRA Activities

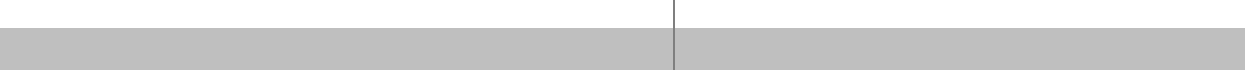

\section{IoT (from IoT doc)}

IoT (from IoT doc)

SP: Authentication 
ISO/IEC 27037:2012

JSR (Java Specification Request) 221 (developed by the Java

Community Process)

W3C XML

W3C Resource Description Framework (RDF)

W3C JavaScript Object Notation (JSON)-LD 1.0

W3C Document Object Model (DOM) Level 1 Specification

W3C XQuery 3.0

W3C XProc

W3C XML Encryption Syntax and Processing Version 1.1

W3C XML Signature Syntax and Processing Version 1.1

SP: Authentication

W3C XPath 3.0

W3C XSL Transformations (XSLT) Version 2.0

W3C Efficient XML Interchange (EXI) Format 1.0 (Second

Edition)

W3C RDF Data Cube Vocabulary

W3C Data Catalog Vocabulary (DCAT)

W3C HTML5 A vocabulary and associated APIs for HTML and

XHTML

W3C Internationalization Tag Set (ITS) 2.0

W3C OWL 2 Web Ontology Language

W3C Platform for Privacy Preferences (P3P) 1.0

W3C Protocol for Web Description Resources (POWDER)

W3C Provenance $\quad$ Defense,

W3C Rule Interchange Format (RIF)

W3C Service Modeling Language (SML) 1.1

W3C Simple Knowledge Organization System Reference

(SKOS)

W3C Simple Object Access Protocol (SOAP) 1.2

W3C SPARQL 1.1

W3C Web Service Description Language (WSDL) 2.0

W3C XML Key Management Specification (XKMS) 2.0 


\section{Standard Name/Number}

OGC $^{\circledR}$ OpenGIS ${ }^{\circledR}$ Catalogue Services Specification 2.0.2 -

ISO Metadata Application Profile

OGC $^{\circledR}$ OpenGIS ${ }^{\circledR}$ GeoAPI

OGC $^{\circledR}$ OpenGIS $^{\circledR}$ GeoSPARQL

OGC $^{\circledR}$ OpenGIS ${ }^{\circledR}$ Geography Markup Language (GML)

Encoding Standard

OGC ${ }^{\circledR}$ Geospatial eXtensible Access Control Markup Language

(GeoXACML) Version 1

OGC $^{\circledR}$ network Common Data Form (netCDF)

OGC ${ }^{\circledR}$ Open Modelling Interface Standard (OpenMI)

OGC $^{\circledR}$ OpenSearch Geo and Time Extensions

OGC $^{\circledR}$ Web Services Context Document (OWS Context)

OGC $^{\circledR}$ Sensor Web Enablement (SWE)

OGC $^{\circledR}$ OpenGIS ${ }^{\circledR}$ Simple Features Access (SFA)

OGC $^{\circledR}$ OpenGIS ${ }^{\circledR}$ Georeferenced Table Joining Service (TJS)

Implementation Standard

OGC $^{\circledR}$ OpenGIS ${ }^{\circledR}$ Web Coverage Processing Service Interface

(WCPS) Standard

OGC $^{\circledR}$ OpenGIS ${ }^{\circledR}$ Web Coverage Service (WCS)

OGC $^{\circledR}$ Web Feature Service (WFS) 2.0 Interface Standard

OGC $^{\circledR}$ OpenGIS ${ }^{\circledR}$ Web Map Service (WMS) Interface Standard

OGC ${ }^{\circledR}$ OpenGIS ${ }^{\circledR}$ Web Processing Service (WPS) Interface

Standard

OASIS AS4 Profile of ebMS 3.0 v1.0

OASIS Advanced Message Queuing Protocol (AMQP) Version

1.0

OASIS Application Vulnerability Description Language

(AVDL) v1.0

OASIS Biometric Identity Assurance Services (BIAS) Simple

Object Access Protocol (SOAP) Profile v1.0

OASIS Content Management Interoperability Services (CMIS)

OASIS Digital Signature Service (DSS)

OASIS Directory Services Markup Language (DSML) v2.0 


\section{Standard Name/Number}

OASIS ebXML Messaging Services

OASIS ebXML RegRep

OASIS ebXML Registry Information Model

OASIS ebXML Registry Services Specification

OASIS eXtensible Access Control Markup Language (XACML)

OASIS Message Queuing Telemetry Transport (MQTT)

OASIS Open Data (OData) Protocol

OASIS Search Web Services (SWS)

OASIS Security Assertion Markup Language (SAML) v2.0

OASIS SOAP-over-UDP (User Datagram Protocol) v1.1

OASIS Solution Deployment Descriptor Specification v1.0

OASIS Symptoms Automation Framework (SAF) Version 1.0

OASIS Topology and Orchestration Specification for Cloud

Applications Version 1.0

OASIS Universal Business Language (UBL) v2.1

OASIS Universal Description, Discovery and Integration

(UDDI) v3.0.2

OASIS Unstructured Information Management Architecture

(UIMA) v1.0

OASIS Unstructured Operation Markup Language (UOML) v1.0

OASIS/W3C WebCGM v2.1

BDAP: Analytics

OASIS Web Services Business Process Execution Language

(WS-BPEL) v2.0

OASIS/W3C - Web Services Distributed Management

(WSDM): Management Using Web Services (MUWS) v1.1

OASIS WSDM: Management of Web Services (MOWS) v1.1

OASIS Web Services Dynamic Discovery (WS-Discovery) v1.1

OASIS Web Services Federation Language (WS-Federation)

v1.2

OASIS Web Services Notification (WSN) v1.3

IETF Simple Network Management Protocol (SNMP) v3

IETF Extensible Provisioning Protocol (EPP) 


\section{Standard Name/Number}

NCPDPD Script standard

ASTM Continuity of Care Record (CCR) message

Healthcare Information Technology Standards Panel (HITSP)

C32 HL7 Continuity of Care Document (CCD)

PMML Predictive Model Markup Language

Add Open Group standards from Information Base,

https://www2.opengroup.org/ogsys/jsp/publications/viewSIB.jsp

Dash7

H.265

VP9

Daala

WebRTC

X.509

MDX

\section{NIEM-HLVA}

NIEM-MPD

NIEM-Code List Specifications

NIEM Conformance Specification

NIEM-CTAS

NIEM-NDR

Non-Normative Guidance in Using NIEM with JSON

DCC Data Package, version 1.0.0-beta.17 (a specification)

released March of 2016

DCC Observ-OM।

DCC PREMIS

DCC PROV

DCC QuDEx

DCC SDMX, specification 2.1 last amended May of 2012

DCC TEI

BDFP: Processing: Stream Processing; BDFP: Processing: Stream Processing; BDFP: Processing: Stream Processing;

Government Operations, Defense, Commercial Government Operations, Defense, Commercial Government Operations, Defense, Commercial Government Operations, Defense, Commercial Government Operations, Defense, Commercial Government Operations, Defense, Commercial

Government Operations, Defense, Commercial

BDAP: collection; BDFP: messaging BDAP: collection; BDFP: messaging BDAP: collection; BDFP: messaging BDAP: collection; BDFP: messaging BDAP: collection; BDFP: messaging BDAP: collection; BDFP: messaging BDAP: collection; BDFP: messaging 


\section{Appendix E: References}

[1] W. Chang and NIST Big Data Public Working Group, "NIST Big Data Interoperability Framework: Volume 1, Definitions (SP1500-1),” 2015.

[2] W. Chang and NIST Big Data Public Working Group, "NIST Big Data Interoperability Framework: Volume 2, Big Data Taxonomies (SP1500-2),” 2015.

[3] W. Chang and NIST Big Data Public Working Group, "NIST Big Data Interoperability Framework: Volume 3, Use Cases and General Requirements (SP1500-3),” 2015.

[4] W. Chang and NIST Big Data Public Working Group, "NIST Big Data Interoperability Framework: Volume 4, Security and Privacy (SP1500-4),” 2015.

[5] W. Chang and NIST Big Data Public Working Group, "NIST Big Data Interoperability Framework: Volume 5, Architectures White Paper Survey (SP1500-5),” 2015.

[6] W. Chang and NIST Big Data Public Working Group, "NIST Big Data Interoperability Framework: Volume 6, Reference Architecture (SP1500-6),” 2015.

[7] W. Chang and NIST Big Data Public Working Group, "NIST Big Data Interoperability Framework: Volume 8, Reference Architecture Interface (SP1500-9),” 2017.

[8] W. Chang and NIST Big Data Public Working Group, "NIST Big Data Interoperability Framework: Volume 9, Adoption and Modernization (SP1500-10),” 2017.

[9] F. Farance, “Adapted from the Refactoring Metadata Status Report,” 2016.

[10] Cloud Security Alliance, "Expanded Top Ten Big Data Security and Privacy Challenges,” Cloud Security Alliance, 2013. [Online]. Available:

https://downloads.cloudsecurityalliance.org/initiatives/bdwg/Expanded_Top_Ten_Big_Data_Secu rity_and_Privacy_Challenges.pdf.

[11] A. DiStefano, K. E. Rudestam, and R. Silverman, Encyclopedia of Distributed Learning, Annotated. SAGE Publications, 2003.

[12] EXECUTIVE OFFICE OF THE PRESIDENT Office of Management and Budget, "Federal Participation in the Development and Use of Voluntary Consensus Standards and in Conformity Assessment Activities,” OMB Circ. A-119, vol. 81 FR 4673, p. 43, 2016.

[13] ISO/IEC JTC 1: Information Technology, “Big Data, Preliminary Report 2014,” 2014.

[14] G. De Simoni and R. Edjlali, “Magic Quadrant for Metadata Management Solutions,” Gart. Repr., pp. 1-26, 2017.

[15] The Library of Congress, “CQL: Contextual Query Language,” Search/Retrival via URL, 2013. [Online]. Available: http://www.loc.gov/standards/sru/cql/contextSets/. [Accessed: 02-Jul-2017].

[16] W3C, “Resource Description Framework (RDF),” Semantic Web, 2014. [Online]. Available: https://www.w3.org/RDF/. [Accessed: 02-Jul-2017].

[17] SAS, "The new data integration landscape: Moving beyond ad hoc ETL to an enterprise data integration strategy.”

[18] K. Cagle, “Understanding the Big Data Life-Cycle,” 2015. [Online]. Available: https://www.linkedin.com/pulse/four-keys-big-data-life-cycle-kurt-cagle. [Accessed: 10-Jun- 
NIST BIG DATA INTEROPERABILITY FRAMEWORK: VOLUME 7, STANDARDS ROADMAP

2017].

[19] W. W. Eckerson, "How to Create a Culture of Governance,” The New BI Leader, 2017. [Online]. Available: https://www.eckerson.com/articles/how-to-create-a-culture-of-governance. [Accessed: 10-Jun-2017].

[20] Kofax, "Integrating Data Sources is an Expensive Challenge for the Financial Services Sector (White Paper),” 2015.

[21] OVUM, “Ovum Decision Matrix: Selecting an Enterprise Mobility Management Solution, 2017$18, ” 2017$. 